\title{
Potentialités Des Agroforêts Contre Les Changements Climatiques En Zone D'écotone Forêt-Savane Du Cameroun
}

\author{
Etchike D. A. B., \\ Laboratoire de Botanique Appliquée de l'Université de Dschang, \\ Dschang, Cameroun
}

Ngassoum M. B.,

Ecole Nationale Supérieure des Sciences Agro-Industrielles de l'Université de Ngaoundéré, Cameroun

Mapongmetsem P. M.,

Laboratoire de Biodiversité et Développement Durable de l'Université de

Ngaoundéré, Ngaoundéré, Cameroun

Doi:10.19044/esj.2020.v16n15p319 URL:http://dx.doi.org/10.19044/esj.2020.v16n15p319

\section{Resume}

L'agroforêt fait partie du paysage agraire dans l'Arrondissement de Ndikiniméki dans la région du centre Cameroun, malheureusement sujette à de nombreuses pressions. En dépit des travaux menés sur les agroforêts du Centre et du Sud Cameroun, peu se sont intéressés à sa contribution dans la lutte contre le réchauffement climatique. L'objectif de la présente étude est de démontrer l'efficacité des agroforêts dans la sécurité alimentaire des populations, la conservation de la phytodiversité et dans la lutte contre les changements climatiques. Pour ce faire une analyse participative a été réalisée avec 74 exploitants d'agroforêts répartis dans cinq villages. Des inventaires botaniques ont été réalisés dans chacune de ces agroforêts. Les données recueillies ont fait l'objet d'analyses diverses (calcul des indices écologiques, de diversité, de biomasse et des tests statistiques). Les agroforêts jeunes (moins de 15 ans) et vieilles (supérieur à 45 ans) sont les plus importantes dans la zone. La classe de superficie supérieure à $1500 \mathrm{~m}^{2}$ vient au premier rang avec $32 \%$. Les cultures vivrières les plus fréquentes dans les agroforêts sont les tubercules (Manihot esculanta), les légumineuses (Phaseolus vulgaris) et le maïs (Zea mays). La cacaoculture est la plus répandue des cultures de rente de la zone avec une fréquence de 0,73. Le safoutier (Dacryodes edulis) et le manguier (Mangifera indica) viennent au premier rang des fruitiers exotiques. La richesse spécifique est évaluée à 72 espèces reparties en 63 genres et 29 familles. Parmi les familles écologiquement importantes nous avons les Moraceae $(\mathrm{FIV}=26,96 \%)$ et Apocynaceae $(\mathrm{FIV}=25,66 \%)$. Les indices de 
diversité de Shannon sont les plus importants dans les agroforêts de Ndokononoho et Oundjock. Mangifera indica (64,80 t/ha) et Dacryodes edulis (41,23 t/ha) sont les espèces qui ont les biomasses les plus importantes de la zone. La biomasse ligneuse totale dans la zone est estimée à 4261,72t pour un stock de carbone total de 2003t, ce qui correspond à un stock total de $\mathrm{CO}_{2}$ de $7351,03 \mathrm{t} \mathrm{CO}_{2}$ qui rapporterait aux exploitants un crédit carbone de 44.026.788,87 Franc CFA. Ces résultats attestent de l'efficacité de ces agroforêts dans la lutte contre le réchauffement climatique et pouvant servir dans l'orientation et l'application des politiques du mécanisme REDD++ sur le plan local et régional.

Mots clés : Agroforêts, phytodiversité, Ndikiniméki, stock de carbone, atténuation, REDD++

\title{
Potential of Agroforests Against Climate Change in the Area of Forest-Savannah Ecotone from Cameroon
}

\author{
Etchike D. A. B., \\ Laboratoire de Botanique Appliquée de l'Université de Dschang, \\ Dschang, Cameroun \\ Ngassoum M. B.,
}

Ecole Nationale Supérieure des Sciences Agro-Industrielles de 1'Université de Ngaoundéré, Cameroun

Mapongmetsem P. M.,

Laboratoire de Biodiversité et Développement Durable de l'Université de

Ngaoundéré, Ngaoundéré, Cameroun

\begin{abstract}
The agroforest is part of the agrarian landscape in the Ndikiniméki suddivision in the central region of Cameroon, unfortunately subject to many pressures. Despite the works carried out on the agroforests of Centre and South regions of Cameroon, few have paid attention to its contribution to the fight against global warming. The objective of this present study is to demonstrate the effectiveness of agroforests in the food security of the populations, the conservation of phytodiversity and in the fight against climate change. To do this, a participatory analysis was carried out with 74 agroforest farmers in five villages. Botanical inventories were carried out in each of these agroforests. The data collected were analyzed in various ways (calculation of ecological,
\end{abstract}


diversity and biomass indices as wells as statistical tests). Young groforests (less than 15 years old) and old (over 45 years old) are the most prominent in the zone. The surface area class exceeding $1500 \mathrm{~m}^{2}$ comes first with $32 \%$. The most common food crops in the agroforests are tubers (Manihot esculanta), legumes (Phaseolus vulgaris) and maize (Zea mays). Cocoa farming is the most common cash crop in the zone with a frequency of 0.73 . Safoutier trees (Dacryodes edulis) and mango trees (Mangifera indica) are ranks first among the exotic fruit trees. Species richness is estimated at 72 species in 63 genera and 29 families. Among the ecologically important families, we have the Moraceae $(\mathrm{FIV}=26.96 \%)$ and Apocynaceae $(\mathrm{FIV}=25.66 \%)$. The Shannon's diversity indices are the most important for the Ndokononoho and Oundjock agroforests. Mangifera indica (64.80 t/ha) and Dacryodes edulis (41.23 t/ha) are the species with the most important biomass in the zone of the study. The total woody biomass in the area is estimated at $4261,72 \mathrm{t}$ for a total carbon stock of 2003t, which corresponds to a total $\mathrm{CO}_{2}$ stock of $7351.03 \mathrm{t} \mathrm{CO}_{2}$ which would bring operators a carbon credit of 44026788.87 Franc CFA. These results justify the effectiveness of these agroforests in the fight against global warming and can be used as a guide and the implementation of the policies of the REDD ++ mechanisms at the local and regional levels.

Keywords: Agroforests, phytodiversity, Ndikiniméki, Carbon stock, Mitigation, REDD ++

\section{Introduction}

La biodiversité est reconnue comme un patrimoine vital commun à toute l'humanité. Seulement, elle est sujette de nos jours à une dégradation et à des transformations engendrées par des formes de mise en valeur de plus en plus incompatibles avec sa préservation (Manfo et al, 2015). Tchotsoua et al. (1998) affirment que ce sont les activités humaines qui sont la cause principale de sa dégradation et de sa destruction vu la démographie croissante observée ces dernières années. De ce fait, l'agriculture itinérante sur brûlis est considérée comme le premier facteur responsable des pertes du couvert forestier (de plus de 70 à $90 \%$ ) au Cameroun (COMIFAC, 2010). De nos jours, avec cet accroissement démographique et les besoins croissants en terres agricoles, la diversité biologique et le nombre d'arbres épargnés lors des défrichements culturaux est en voie de régression rapide parce que soumise à de grandes pressions anthropiques et naturelles désignées sous le nom collectif de changements climatiques (GIEC, 2002; Mapongmetsem et al., 2016). Les changements climatiques et leurs impacts sont désormais reconnus comme l'un des plus grands défis du monde, de ses peuples, de son environnement et de ses économies (GIEC, 2007). Ces changements entraîneront également une perte de biodiversité. 15 à $37 \%$ des plantes et espèces animales terrestres 
pourraient disparaître d'ici à 2050 (FIDA, 2008). L'accroissement de la fréquence et de l'ampleur des phénomènes météorologiques extrêmes comme les sécheresses, les inondations ou les tempêtes : conséquences directes des changements climatiques, sont déjà une réalité. La santé, les écosystèmes terrestres et aquatiques et les systèmes socio-économiques comme l'agriculture, l'exploitation forestière, la pêche et les ressources en eau, éléments essentiels au développement subissent déjà les effets induits des changements climatiques (GIEC, 2007).

La lutte contre ces aléas climatiques constitue aujourd'hui l'une des préoccupations majeures pour les gouvernements et en particulier celui du Cameroun compte tenu de l'importance de son impact sur la vie des populations (UICN-PC, 2013). Depuis la décennie 90, les systèmes d'exploitation agricoles en dépit de l'augmentation des surfaces cultivées, se sont avérés peu productifs et incapables de satisfaire les besoins de nutrition d'une population de plus en plus croissante comme celle du Cameroun (Manfo et al., 2015). Cette situation plonge de plus en plus la population dans une sous-alimentation. Au vu de tout ceci, les systèmes agroforestiers dans le monde se présentent aujourd'hui à la fois comme une alternative de conservation de la phytodiversité et de satisfaction des besoins socioéconomiques des populations (Manfo et al., 2015 ; Mapongmetsem et al., 2002 ; Mapongmetsem, 2017). Depuis les temps immémoriaux, de nombreuses générations ont utilisé conjointement les ressources agricoles et forestières pour s'assurer une qualité de vie adéquate et créer un cadre de vie agréable (De Baets et al., 2007). Dans l'écotone forêt-savane de l'Arrondissement de Ndikiniméki dans le Département du Mbam et Inoubou, le paysage rural, plus ou moins converti en parcelles cultivées est dominé par divers systèmes agroforestiers parmi lesquels les agroforêts. Les agroforêts se caractérisent généralement par un peuplement dominant, principale source de revenu ou d'utilisation (hévéa, caféier, cacaoyer, etc.), tout en étant constituées de nombreux autres composants (arbres, lianes, arbustes), tant en espèces qu'en fréquences, organisés en plusieurs strates (Jagoret, 2011). La culture du cacao est faite sous couvert arboré, les agriculteurs conservant ou introduisant dans leurs plantations des espèces ligneuses utiles (Saj et al., 2013 ; Temgoua et al., 2018). Ces agroforêts en effet, sont conservatrices d'un certain niveau de biodiversité, car s'apparente à celle des forêts et pouvant aller jusqu'à un niveau proche de celui de la forêt secondaire selon les systèmes (Sonwa et al., 2007 ; Tayo, 2014 ; Saj et al., 2017).

Dans cet écotone forêt-savane, les agroforêts qui en font partie n'ont pas encore été évalués quant à leurs contributions dans la lutte contre le réchauffement climatique. L'objectif de la présente étude est de démontrer non seulement l'importance des agroforêts dans la conservation et la sécurité alimentaire des populations dans cet écotone, mais également son efficacité 
dans l'adaptation, l'atténuation du réchauffement climatique. Il s'agira spécifiquement d'inventorier les différentes espèces cultivées et locales misent en association dans ces agroforêts et de quantifier le stock de carbone accumulé par la biomasse des arbres inventoriés dans ces agrosystèmes.

\section{Matériels et méthodes}

\section{Localisation du site d'étude}

Notre choix s'est porté sur l'arrondissements de Ndikiniméki dans le Mbam et Inoubou tout d'abord pour la pertinence et la complexité actuelle de ses mosaïques forêts-savanes et des activités agrosylvopastorales menées dans la région (Fig.1). Par ailleurs sur la répartition de ses formations végétales partant de l'est du massif forestier du confluent du Mbam et du Kim jusqu'à la hauteur de la localité de Ntui (Youta, 1998). Ndikiniméki s'étend de $4^{\circ} 28^{\prime}$ à $4^{\circ} 70^{\prime}$ de latitude Nord et de $10^{\circ} 28^{\prime}$ à $11^{\circ} 00^{\prime}$ de longitude Est (Kengne et Ali, 2002). Il y règne un climat de type équatorial à deux saisons de pluies entrecoupées de deux saisons sèches, avec une température qui se stabilise autour de $25^{\circ} \mathrm{C}$. La pluviométrie moyenne annuelle est de $1440 \mathrm{~mm}$. Les moyennes thermiques sont comprises entre 20 et 25 degré (Soh, 1999). Le relief de la zone est constitué de deux ensembles de plateaux de hautes et basses altitudes. Les hauts plateaux dont l'altitude varie entre 600 et 1000 mètres sont établis au centre et à l'Est de la zone, tandis que le bas plateau occupe la partie Ouest de la zone avec des altitudes inférieures à 500 mètres (Bohin, 1992 cit. Soh, 1999). Les sols de cette région sont de type ferralitique rouge et jaune (sols argilo-sableux). La végétation est représentée d'une part par une forêt secondaire peuplée des grands arbres tels que Ceiba pentadra, Albizia grandifolia, Mansonia altissima, Musanga cecropioides, Adansonia digitata, Ricinodendron heudolotii, Celtis tessmanii, Sterculia oblonga, Celtis zenkeri (Letouzey, 1985 ; Zapfack et al., 2000). Des jachères dominées par Chromolaena odorata, Pennisetum purpureum, Imperata cylindrica, Costus afer et Ageratum sp. sont caractéristiques de la zone (Achoundong et al., 1996a, 1996b). 

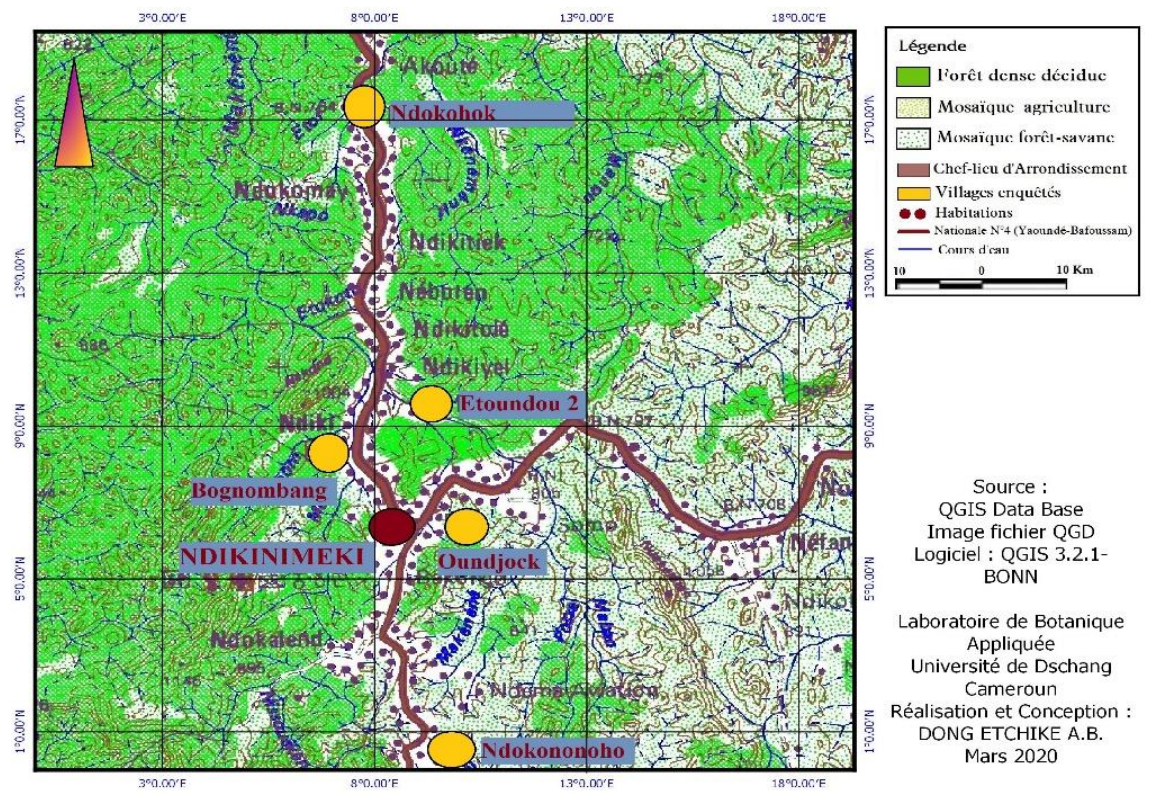

Figure 1 : Cartes du site d'étude : Arrondissement de Ndikiniméki.

\section{Méthodes et outils}

L'approche méthodologique adoptée est participative et réitérative. Pour ce faire des informations socio-économiques et agro-écologiques sur la gestion des systèmes agroforestiers ont été collectées. Les chefs d'exploitations ont été enquêtés avec l'aide des agents de services forestiers et agricoles agrées. Un taux de sondage de 5,33\% (74 enquêtés sur une population de 1386) a été adopté sur la base des statistiques du MINADER (2005) qui indique par ailleurs la faible population de la zone (moins de 5 habitants au $\mathrm{km}^{2}$ ). Les enquêtes ethnobotaniques se sont faites par des interviews semi- structurées individuelles au moyen d'un questionnaire comportant des questions ouvertes et des questions fermées suivant la méthode de Mapongmetsem et al. (2016). Un échantillon de 74 exploitants d'agroforêts a été interrogé dans cinq villages de l'Arrondissement de Ndikiniméki à savoir : Bognombang, Etoundou 2, Oundjock, Ndokohok et Ndokononoho, le tout sur une superficie totale de $136.625 \mathrm{~m}^{2}$ soit 13,66 ha. S'agissant des inventaires botaniques, tous les arbres dont le diamètre du tronc à hauteur de poitrine (dbh) mesuré à $1,3 \mathrm{~m}$ du sol pour les arbres sans contreforts et sans anomalies) était supérieur ou égal à $8 \mathrm{~cm}$ ont été identifiés et mesurés suivant les recommandations de White et Edwards (2001). La nomenclature botanique utilisée est celle adoptée par Lebrun \& Stork (1991-1997) afin de pouvoir comparer les résultats avec la bibliographie. Divers indices écologiques ont été déterminés, permettant d'étudier la structure des peuplements et d'avoir une évaluation quantitative de la diversité du peuplement. Il s'agit du calcul 
des indices de Shannon-Weaver et de Simpson. Les calculs des densités, de la dominance et des fréquences relatives ont également été effectués pour mieux apprécier l'importance écologique des espèces et des familles, précisément par le calcul de l'Importance Value Index (IVI) et du Family Importance Value (FIV).

L'indice de Shannon et Weaver (Sonké, 1998) qui est un indice d'abondance basé sur la diversité spécifique qui permet de décrire avec d'autres indices la structure d'une communauté.

\section{ISH $=-\sum((\mathrm{Ni} / \mathrm{N}) * \log 2(\mathrm{Ni} / \mathrm{N}))$ ou ISH $=-$ Somme de i=1 à s (pi * log2 \\ pi)}

L'indice de Simpson mesure la probabilité que deux individus sélectionnés au hasard appartiennent à la même espèce.

$$
\mathbf{D}=\sum \mathbf{N i}(\mathbf{N i}-1) / \mathbf{N}(\mathbf{N}-1)
$$

Densité relative $=\sum(\mathbf{N i} / \mathbf{N}) * \mathbf{1 0 0}$ (Traoré, 1997)

$\mathbf{N i}=$ Nombre d'individus de l'espèce à l'hectare et $\mathbf{N}=$ Nombre total d'individus à l'hectare

Dominance relative $=\sum(\mathbf{N i} / \mathbf{N}) * \mathbf{1 0 0}($ White \& Edwards, 2001).

$\mathbf{N i}=$ Valeur absolue de la dominance de l'espèce à l'échelle de la population et $\mathbf{N}=$ Somme des valeurs de la dominance de toutes les espèces à l'échelle de la population.

Fréquence relative $=\sum(\mathbf{N i} / \mathbf{N}) * \mathbf{1 0 0}$ (Sonké, 2004)

$\mathbf{N i}=$ Valeur absolue de la fréquence de l'espèce à l'échelle de la population et $\mathbf{N}=$ Somme des valeurs des fréquences de toutes les espèces à l'échelle de la population.

L'Importance Value Index (IVI) a été calculée suivant la formule qui intègre la densité, la dominance et la fréquence relative des espèces. Cet index permet de mieux apprécier l'importance des espèces des communautés végétales (Gonmadjé et al. (2011, 2012) ; Kacholi, 2014).

IVI $=$ Densité relative + Dominance relative + Fréquence relative.

Le Family Importance Value (FIV) apprécie mieux l'importance des familles dans une communauté végétale.

FIV = Densité relative + Dominance relative + Diversité relative.

Le calcul de la biomasse aérienne des arbres s'est fait selon la méthode non destructive qui consiste en une évaluation de la biomasse de l'arbre à partir de son diamètre. Cette biomasse a été estimée à l'aide des équations allométriques de Ngomanda et al. (2013, 2014) spécifiques aux forêts du bassin du Congo. Parmi ces modèles, celui qui tient compte du diamètre et de la densité de l'espèce et qui s'écrit comme suit :

$$
Y=\exp \left[-4,0596+4,0624 \ln D-0,228(\ln D)^{2}+1,4307 \ln \rho\right]
$$

Où Y s'exprime en $\mathrm{kg}$ ou (t), D en cm, et $\boldsymbol{\rho}=0,69826 \mathrm{~g} / \mathrm{cm}$.

La fraction de carbone représente $47 \%$ de la biomasse sèche de l'arbre. Pour avoir le stock de carbone (Sc) accumulé, la biomasse (Y) a été multipliée par 
0,47 suivant la formule ci-après (GIEC, 2006 ; Tsoumou et al., 2016) :

$$
\mathbf{S c}=\mathbf{0 , 4 7} * \mathbf{Y}
$$

Où Sc s'exprime en tonne carbone (tC). Le stock de $\mathrm{CO}_{2}$ atmosphérique équivalent est estimé en multipliant le stock de carbone issu de la biomasse par 3,67.

La biomasse des palmiers a été calculée suivant la formule ci-contre (Winrock, 2005) :

$$
Y=23,487+41,851 * \ln (H)^{2}
$$

\section{Analyses des données}

Les données collectées ont fait l'objet d'analyses diverses en l'occurrence des analyses floristiques via les calculs de paramètres écologiques (IVI, FIV) et les indices de diversités écologiques (ShannonWeaver, Simpson, Evenness, Equitabilité J de Piélou, Fisher alpha, Raréfaction). Par ailleurs des analyses et calculs de biomasses ligneuses et du stock de carbone pour une meilleurs caractérisation de la flore dans ces agroforêts et leurs efficacités dans l'accumulation du carbone. Des tests univariés (ANOVA à un seuil de probabilité de 5\%) ont été effectués sur plusieurs paramètres pour déterminer les différences significatives $\left(\mathrm{H}_{0}\right.$ indiquant l'égalité et $\mathrm{H}_{1}$ indiquant au moins une différence). Lorsqu'une différence significative est observée, nous complétons l'ANOVA par des comparaisons multiples (Dagnelie, 1998) en effectuant les tests de Duncan et/ou de Tukey pour ressortir les plus petites différences significatives (ppds). Les outils utilisés pour ces analyses sont entre autres le tableurs Office Excel (Windows 10) et Office Excel/Numbers (Macintosh), XLStat (5.03), le logiciel R, RStudio (3.5, 1.1.4) et PAST (3.0).

\section{Résultats et discussion}

\section{Caractéristiques socio-économiques, biophysiques et fonctionnelles des agroforêts}

Les enquêtes ethnobotaniques au sein des villages ont révélé diverses appellations des agroforêts par les populations locales. Parmi les communautés rencontrées l'on a les anglophones qui utilisent l'expression « House's farm » ou « Farm house » pour désigner l'agroforêt, ce qui signifie littéralement «champs de maison». Signification similaire pour les expressions « Mohwong me mèerh » chez les Bafia, « M'benla » en kinding, « Nn'a tchounda » en Bantoum. Les exploitants de Tonga les appellent « Nn'a lack», tandis que « N'ka » est l'expression utilisée par les exploitants Bouda, « Topotopo yombell » par les Banen, «N'kilien ékô »par les Nyokon et « Manyènyè » par les exploitants Yambassa (Tableau 1). La majorité de ces exploitants sinon tous mentionnent que ce type d'exploitation est en quelque sorte « un mélange de tout » ou encore désigné «Vergers» par certains. 
Tableau 1 : Noms vernaculaires des types d'exploitations.

\begin{tabular}{|c|c|c|c|}
\hline Tributs & Pourcentage & Champs & Agroforêt \\
\hline Anglophone & 5,4 & Farm & $\begin{array}{c}\text { House's farm / Farm } \\
\text { house }\end{array}$ \\
\hline Bafia & 2,7 & Dème & $\begin{array}{l}\text { Dème à bedilak } \\
\text { Mohwong me mèerh }\end{array}$ \\
\hline Bamiléké & 9,45 & Tuèck & $\begin{array}{c}\text { M'benla (kinding) } \\
\text { Nn'a tchounda (Bantoum) }\end{array}$ \\
\hline & & Nn'a & $\begin{array}{c}\text { Nn'a lack (Tonga) } \\
\text { N'ka (Bouda) }\end{array}$ \\
\hline Banen & 66,21 & Embom & $\begin{array}{l}\text { Embom ayombell } \\
\text { Topotopo yombell }\end{array}$ \\
\hline Nyokon & 1,4 & N'kilien & N'kilien ékô \\
\hline Yambassa & 14,86 & N'tèmè & Manyènyè \\
\hline
\end{tabular}

Les agroforêts jeunes (moins de 15 ans) et âgées (plus de 45 ans) sont les plus importantes dans la zone avec un taux moyen de 62,5\% (31,42\% + $31,08 \%)$. Les tranches 16-30 ans et 31-45 ans viennent respectivement en troisième et quatrième rang avec des taux moyen respectifs de $18,98 \%$ et $18,42 \%$ (Tableau 2). L'analyse de variance ne montre pas une différence significative entre les 4 tranches d'âges d'agroforêts (colonnes) d'une part et entre les 5 localités (lignes). La variation observée n'est qu'apparente entre les âges de ces agroforêts.

Parlant de la superficie de ces agroforêts, il ressort des investigations que la classe de superficie supérieure à $1500 \mathrm{~m}^{2}$ vient au premier rang avec $32 \%$ suivie de la classe inférieure à $500 \mathrm{~m}^{2}$ avec $25,9 \%$. En troisième position les agroforêts avec un taux de $20,1 \%$ pour les superficies comprises entre 500 et $1000 \mathrm{~m}^{2}$ suivent. Les agroforêts de la classe 1001 à $1500 \mathrm{~m}^{2}$ sont les moins représentées $(4,5 \%)$. Il est important de relever le taux assez élevé $(17,26 \%)$ des exploitants ne sachant pas avec exactitude la superficie ou les limites de leur exploitation. L'analyse de variance montre une différence significative $(\mathrm{P}<0,05)$ entre les 4 tranches de superficie d'agroforêts (colonnes). La tranches de superficie 1001 à $1500 \mathrm{~m}^{2}$ varie très significativement (a) par rapport aux tranches $<500 \mathrm{~m}^{2}(\mathrm{~b})$ et $>1500 \mathrm{~m}^{2}(\mathrm{~b})$. 
Tableau 2 : Répartition des pourcentages par classe de l'âge et de la superficie des agroforêts.

\begin{tabular}{|c|c|c|c|c|c|c|c|c|}
\hline \multirow{2}{*}{ Villages } & \multicolumn{4}{|c|}{ Ages des agroforêts } & \multicolumn{4}{|c|}{ Superficies des agroforêts (m²) } \\
\hline & $<15$ & $16-30$ & $31-45$ & $>45$ & $<500$ & $\begin{array}{l}500- \\
1000\end{array}$ & $\begin{array}{c}1001 \text { - } \\
1500\end{array}$ & $>1500$ \\
\hline Bognombang & $16,6 \mathrm{a}$ & $41,6 a$ & $16,6 \mathrm{a}$ & $25 \mathrm{a}$ & $16,6 b$ & $33,3 \mathrm{ab}$ & $0 \mathrm{a}$ & $0 \mathrm{~b}$ \\
\hline Etoundou 2 & $12,5 \mathrm{a}$ & $12,5 \mathrm{a}$ & $37,5 \mathrm{a}$ & $37,5 \mathrm{a}$ & $25 b$ & $12,5 \mathrm{ab}$ & $12,5 \mathrm{a}$ & $37,5 b$ \\
\hline Oundjock & $80 \mathrm{a}$ & $10 \mathrm{a}$ & $10 \mathrm{a}$ & $0 \mathrm{a}$ & $50 \mathrm{~b}$ & $20 \mathrm{ab}$ & $0 \mathrm{a}$ & $20 b$ \\
\hline Ndokohok & $21,4 \mathrm{a}$ & $14,2 \mathrm{a}$ & $21,4 \mathrm{a}$ & $42,9 a$ & $21,4 b$ & $21,4 \mathrm{ab}$ & $7,1 \mathrm{a}$ & $42,8 b$ \\
\hline Ndokononoho & $26,6 a$ & $16,6 \mathrm{a}$ & $6,6 a$ & $50 \mathrm{a}$ & $16,6 b$ & $13,3 \mathrm{ab}$ & $3,3 \mathrm{a}$ & $60 b$ \\
\hline Moyenne \pm ET & $\begin{array}{c}31,42 \pm \\
27,7\end{array}$ & $\begin{array}{c}18,98 \pm \\
12,9\end{array}$ & $\begin{array}{c}18,42 \pm \\
12,1\end{array}$ & $\begin{array}{c}31,08 \pm \\
19,6\end{array}$ & $\begin{array}{c}25,92 \pm \\
13,90\end{array}$ & $\begin{array}{l}20,1 \pm \\
8,40\end{array}$ & $\begin{array}{c}4,58 \pm \\
5,3\end{array}$ & $32,06 \pm 22,9$ \\
\hline Probabilité & \multicolumn{4}{|c|}{$0,0005 * * *$} & \multicolumn{4}{|c|}{$0,0001 * * *$} \\
\hline
\end{tabular}

Légende : Moyenne \pm écarts types (ET). Séparation des moyennes par le test de Tukey à $p=$ 0,05. Les moyennes des taux par colonnes suivies des mêmes lettres ne sont pas significativement différentes. *** niveau de significativité.

\section{Cultures vivrières et cultures de rente}

Les cultures vivrières constituent l'une des principales composantes de ce système traditionnel. Ces exploitations familiales présentent une grande richesse biologique. Cette diversité varie suivant les régimes alimentaires des paysans. Les cultures les plus fréquentes dans les agroforêts sont les tubercules : Manihot esculanta (81,8\%), Ipomoea batatas (76\%) et Xanthosoma sagittifolium (66,8\%) (Tableau 3). La principale légumineuse cultivée est le haricot commun (Phaseolus vulgaris) et ses nombreuses sous-variétés avec $63,4 \%$. Zea mays (61\%) principale céréale cultivée de la famille des Poaceae donne une certaine originalité aux agroforêts de la zone. En effet, le maïs et le manioc constituent avec les gibiers, la base de l'alimentation des populations de la zone. Les produits maraîchers, l'igname (Dioscorea spp.) et l'arachide (Arachis hypogaea) sont fréquents avec respectivement 54,2\%,53,4\% et $44,6 \%$. Les cultures maraîchères pratiquées sont entre autres Lycopersicon esculentum (tomate), Brassica oleracea (Choux), Lactuca sativa (salade) et Daucus carotta (carotte). Diverses autres cultures sont également rencontrées $(30,4 \%)$. Cette dernière catégorie regroupe entre autres le soja (Glycine max), le taro (Colocasia esculenta), la pomme de terre (Solanum tuberrosum), le 
melon (Cucumis melo), la pastèque (Citrullus lanatus) et le concombre ou pistache (Citullus sp.).

Tableau 3 : Taux de la production végétale dans les agroforêts de Ndikiniméki.

\begin{tabular}{c|ccccc|c}
\hline Cultures & Bognombang & $\begin{array}{c}\text { Etoudou } \\
\mathbf{2}\end{array}$ & Oundjock & Ndokohok & Ndokononoho & $\begin{array}{c}\text { Taux (\%) } \pm \\
\text { ET }\end{array}$ \\
\hline Zea mays & 0,66 & 0,75 & 0,9 & 0,71 & 0,03 & $\mathbf{6 1} \pm \mathbf{3 3 , 6}$ \\
Arachis hypogaea & 0,5 & 0,25 & 0,4 & 0,42 & 0,66 & $\mathbf{4 4 , 6} \pm \mathbf{1 5}$ \\
Phaseolus vulgaris & 0,5 & 0,62 & 0,8 & 0,92 & 0,33 & $\mathbf{6 3 , 4} \pm \mathbf{2 3 , 4}$ \\
Manihot esculanta & 0,58 & 1 & 0,9 & 0,71 & 0,9 & $\mathbf{8 1 , 8} \pm \mathbf{1 7}$ \\
Xanthosoma & 0,75 & 0,87 & 0,6 & 0,42 & 0,7 & $\mathbf{6 6 , 8} \pm \mathbf{1 7}$ \\
sagittifolium & 0,75 & 0,87 & 0,6 & 0,85 & 0,73 & $\mathbf{7 6} \pm \mathbf{1 0 , 8}$ \\
Ipomoea batatas & 0,5 & 0,62 & 0,7 & 0,42 & 0,43 & $\mathbf{5 3 , 4} \pm \mathbf{1 2 , 2}$ \\
Dioscorea spp. & 0,5 & 0,5 & 0,8 & 0,85 & 0,06 & $\mathbf{5 4 , 2} \pm \mathbf{3 1 , 5}$ \\
Maraîchérs & 0,41 & 0,37 & 0,3 & 0,21 & 0,23 & $\mathbf{3 0 , 4} \pm \mathbf{8 , 6}$ \\
\hline Autres & \multicolumn{5}{c}{ P-value = 0,516>0,05 } \\
\hline Probabilité & \multicolumn{7}{c}{}
\end{tabular}

L'analyse de variance montre que cette productivité ne varie pas significativement $(\mathrm{P}>0,05)$ entre les 5 localités (colonnes). Il est important de souligner la perception des producteurs enquêtés qui ont signalé des phénomènes climatiques dans la zone comme la baisse de la pluviométrie, le décalage de la grande saison des pluies dû aux retards observés ces dernières années dans son démarrage. Ils relèvent aussi une concentration de la pluie en une courte période, une rupture précoce des pluies à la fin de la petite saison des pluies, une forte occurrence des vents violents et l'augmentation de la chaleur en intensité et en durée (Ogouwalé, 2004; Agossou, 2008). Ces péjorations climatiques ont de fortes conséquences sur les cultures et les calendriers agricoles. Ces cultures supportent mal, aussi bien les retards/ruptures de pluies que les excès de pluie. En effet, le maïs est une espèce exigeante en eau : la période la plus critique à cet égard se situe au moment de la floraison. En effet, ces facteurs induisent des pertes de rendement et de récolte, mais aussi la mauvaise qualité des produits et d'énormes pertes au stockage. Ces facteurs se manifestent différemment selon la situation topo séquentielle de la parcelle (Ogouwalé, 2006).

Pour s'adapter aux changements climatiques, les paysans optent pour la diversification des cultures, la collecte et la commercialisation des ressources forestières, en l'occurrence les Produits Forestiers Non Ligneux (PFNL) à savoir: les fruits, les écorces, les racines, les lianes et les feuilles qui constituent pour de nombreuses familles des sources de médicaments (Mapongmetsem, 2017). Le charbon de bois, la sève de palmier pour la fabrication du vin de palme, les tanins et rotin, les bambous, les chenilles, champignons, latex, gibier et oiseaux sont également cités parmi ces produits dérivés de forêts. Les exploitants les vendent en plus du surplus de la récolte 
pour s'acheter ceux qu'ils ne produisent pas et bien d'autres produits utiles aux ménages. Ces résultats corroborent ceux de Mbolo (1998) à d'Edea dans le Littoral, Dounias et al. (1996c), Sonwa et al. (2002) et Temgoua (2007) dans le Centre et le Sud Cameroun.

Concernant les cultures de rente, il ressort que la cacaoculture est la plus répandue avec une fréquence de $73 \%$. On note que $72,97 \%$ des paysans enquêtés possèdent au moins une cacaoyère. La fréquence de possession (par les paysans) des bananeraies occupe le second rang (45\%) suivie de la caféiculture avec 32\%. Les paysans enquêtés dans la zone ont une faible préférence $(7 \%)$ pour la création de palmeraies (Tableau 4). Ces agroforêts de cacaoyers, de bananiers, de caféiers et de palmiers participent au système de subsistance traditionnelle et répondent aux mêmes motivations de diversifier la production. Ces cultures sont présentes à divers endroits de l'agroforêt en tant que fruitiers et non en tant que féculents comme Musa paradisiaca (bananiers plantains) (Dounias, 1996a).

Tableau 4 : Types de cultures de rente et fréquences de possession dans les villages de Ndikiniméki.

\begin{tabular}{ccccccc}
\hline $\begin{array}{c}\text { Type de } \\
\text { culture }\end{array}$ & $\begin{array}{c}\text { Bognomban } \\
\text { g: /12hab }\end{array}$ & $\begin{array}{c}\text { Etoudo } \\
\text { u 2: } \\
\text { /8hab }\end{array}$ & $\begin{array}{c}\text { Oundjoc } \\
\text { k: /10hab }\end{array}$ & $\begin{array}{c}\text { Ndokoho } \\
\text { k: /14hab }\end{array}$ & $\begin{array}{c}\text { Ndokononoh } \\
\text { o: /30hab }\end{array}$ & $\begin{array}{c}\text { Total } \pm \\
\text { ET }(\%) \\
\text { /74hab }\end{array}$ \\
\hline Cacaoyère & 0,08 & 0,07 & 0,08 & 0,18 & 0,32 & $\mathbf{7 3} \pm \mathbf{1 0 , 7}$ \\
Caféière & 0 & 0,03 & 0,01 & 0,09 & 0,19 & $\mathbf{3 2} \pm \mathbf{7 , 8}$ \\
$\begin{array}{c}\text { Bananerai } \\
\text { e }\end{array}$ & 0,05 & 0,05 & 0,07 & 0,05 & 0,22 & $\mathbf{4 5} \pm \mathbf{7 , 4}$ \\
Palmeraie & 0,01 & 0 & 0 & 0 & 0,04 & $\mathbf{5} \pm \mathbf{1 , 7}$ \\
\hline
\end{tabular}

Légende : hab=habitant/paysans enquêtés

La cacaoculture au-delà des autres cultures de rente est demeurée et continue d'être la source de revenus la plus fiable pour les paysans de Ndikiniméki. Ces cultures de rente sont soit imbriquées ou en continuité avec les forêts naturelles de proximité des maisons, soit situées plus loin dans les brousses (Jiofack et al., 2013). Ces cultures subissent également les affres des changements climatiques, en l'occurrence la pourriture des fruits sur pieds et les problèmes de séchage des graines et de ces produits pour leur stockage. Pendant ces périodes de pluies, les temps ensoleillés sont très rares et cela empêche le séchage des produits à la maison. Aussi, les pluies « intempestives » qui tombent de façon intermittente viennent de façon spéciale mouiller les produits mis au séchage (Ogouwalé, 2004; Agossou, 2008).

\section{Espèces ligneuses et importance écologique}

Le safoutier (Dacryodes edulis) vient au premier rang des fruitiers exotiques rencontrés dans les agroforêts de Ndikiniméki avec une fréquence moyenne de 12,4\%. Il est suivi du manguier (Mangifera indica), des subligneux bananiers fruits (Musa sapientum), bananiers plantains (Musa 
paradisiaca) et du palmier à huile (Elaeis guineensis) (Tableau 5). Ensuite vient l'oranger commun (Citrus sinensis) avec 9\%, suivie du papayer (Carica papaya) 8,6\%. Le Citronnier (Citrus aurantifolia) et le Corossolier (Annona muricata) viennent avec chacune 6,4\%. L'analyse de variance montre globalement une différence significative entre les fréquences de ces espèces fruitières dans ces différentes agroforêts $(\mathrm{P}<0,05)$. Ces fréquences des espèces fruitières sont significatives entre les localités Etoundou 2 (a), Ndokohok (b) et Ndokononoho (c).

Tableau 5 : Fréquences relatives des espèces fruitières dans les agroforêts de Ndikiniméki.

\begin{tabular}{|c|c|c|c|c|c|c|c|c|}
\hline $\begin{array}{l}\text { Noms } \\
\text { communs }\end{array}$ & $\begin{array}{c}\text { Noms } \\
\text { Scientifiques }\end{array}$ & BOGN & ETOU2 & OUND & NHOK & NDHO & $\begin{array}{c}\text { Frq } \\
\text { Totale }\end{array}$ & $\begin{array}{c}\text { Frq } \\
\text { Moyenne } \\
\pm \text { ET }\end{array}$ \\
\hline Safoutier & Dacryodes edulis & 11 & 8 & 8 & 10 & 25 & 62 & $12,4 \pm 6,2$ \\
\hline Manguier & Mangifera indica & $10 \mathrm{ab}$ & $7 a$ & $7 \mathrm{ab}$ & $13 b$ & $21 c$ & 58 & $11,6 \pm 5,8$ \\
\hline Bananier & Musa spp & $9 a b$ & $7 \mathrm{a}$ & $6 a b$ & $12 \mathrm{~b}$ & $22 \mathrm{c}$ & 56 & $11,2 \pm 6,5$ \\
\hline Palmier & Elaeis guineensis & $9 a b$ & $7 \mathrm{a}$ & $5 a b$ & $13 b$ & $22 c$ & 56 & $11,2 \pm 6,7$ \\
\hline Oranger & Citrus sinensis & $2 \mathrm{ab}$ & $0 \mathrm{a}$ & $6 a b$ & $11 \mathrm{~b}$ & $17 \mathrm{c}$ & 36 & $9 \pm 6,5$ \\
\hline Papayer & Carica papaya & $6 a b$ & $4 a$ & $6 a b$ & $6 b$ & $21 \mathrm{c}$ & 43 & $8,6 \pm 7$ \\
\hline Citronier & $\begin{array}{c}\text { Citrus } \\
\text { aurantifolia }\end{array}$ & $3 a b$ & $2 \mathrm{a}$ & $6 a b$ & $5 b$ & $16 \mathrm{c}$ & 32 & $6,4 \pm 5,6$ \\
\hline Corosolier & Annona muricata & $8 a b$ & $5 a$ & $2 \mathrm{ab}$ & $6 b$ & $11 \mathrm{c}$ & 32 & $6,4 \pm 3,4$ \\
\hline Casmanguier & $\begin{array}{l}\text { Spondias } \\
\text { cytherea }\end{array}$ & $5 \mathrm{ab}$ & $2 \mathrm{a}$ & $5 \mathrm{ab}$ & $3 b$ & $12 \mathrm{c}$ & 27 & $5,4 \pm 3,9$ \\
\hline Goyavier & Psidium guajava & $5 \mathrm{ab}$ & $2 \mathrm{a}$ & $4 a b$ & $5 b$ & $8 \mathrm{c}$ & 24 & $4,8 \pm 2,2$ \\
\hline Mandarinier & Citrus reticulata & $2 \mathrm{ab}$ & $2 \mathrm{a}$ & $5 \mathrm{ab}$ & $3 b$ & $11 \mathrm{c}$ & 23 & $4,6 \pm 3,8$ \\
\hline Avocatier & $\begin{array}{c}\text { Persea } \\
\text { americana }\end{array}$ & $4 a b$ & $2 \mathrm{a}$ & $6 a b$ & $7 b$ & $2 c$ & 21 & $4,2 \pm 2,3$ \\
\hline Cocotier & Cocos nucifera & $3 a b$ & $1 \mathrm{a}$ & $4 a b$ & $6 b$ & $5 c$ & 19 & $3,8 \pm 1,9$ \\
\hline Cœur de bœuf & Annona reticulata & $4 a b$ & $4 a$ & $3 a b$ & $2 b$ & $6 c$ & 19 & $3,8 \pm 1,5$ \\
\hline Pamplemousier & Citrus grandis & $1 \mathrm{ab}$ & $2 \mathrm{a}$ & $1 \mathrm{ab}$ & $3 b$ & $4 c$ & 11 & $2,2 \pm 1,3$ \\
\hline \multicolumn{2}{|c|}{ Probabilité } & $\begin{array}{c}< \\
0,0001\end{array}$ & $<0,003$ & $\stackrel{<}{<, 0001}$ & $\begin{array}{c}< \\
0,0001\end{array}$ & $\stackrel{<}{0,0001}$ & \multicolumn{2}{|c|}{$<0,0001 * * *$} \\
\hline
\end{tabular}

Légende : Frq = Fréquence ; Moyenne \pm écart type (ET). Séparation des moyennes par le test de Duncan à $p=0,05$. Les valeurs de colonnes de mêmes lettres ne sont pas significativement différentes. $* * *$ niveau de significativité globale. Localités : BOGN= Bognombang; ETOU2= Etoundou 2; OUND= Oundjock; $N H O K=$ Ndokohok; $N D H O=$ Ndokononoho

Il ressort que le genre «Citrus » dans son ensemble est non seulement parmi les plus fréquents mais également le plus diversifié de ces fruitiers avec quatre espèces et une nette prédominance de l'oranger commun (Citrus sinensis). La fréquence des fruitiers comme Cocos nucifera, Spondias cytherea et Annona spp. sont également non négligeables dans ces agroforêts et restent assez appréciés aussi bien par les adultes que par les enfants. Certains fruitiers bien que faiblement représentés sont appréciés par les populations locales en particulier par les enfants. C'est le cas de Richardella nervosa (fruit 
œuf), l'arbre à pain Artocarpus altilis (Motobô en Bafia) et Artocarpus heterophyllus (Jacquier).

Les espèces locales associées dans ces agroforêts qui présentent l'Importance Value Index (IVI) les plus importantes sont Senna alata (204,42\%), Voacanga spp (132,46\%), Terminalia glaucescens (84,25\%), Milicia excelsa (74,58\%) et Albizia adianthifolia (61,39\%) (Tableau 6). L'analyse de variance montre globalement une différence significative entre les IVI de ces espèces locales dans ces différentes agroforêts $(\mathrm{P}<0,05)$. Cette variation est apparente dans la localité de Ndokononoho $(\mathrm{P}=0,176)$.

Tableau 6 : Paramètres quantitatifs de caractérisation et Importance Value Index (IVI) des espèces ligneuses locales les plus fréquentes dans les agroforêts de Ndikiniméki.

\begin{tabular}{|c|c|c|c|c|c|c|c|c|}
\hline $\mathbf{N}^{\circ}$ & Espèces & BOGN & ETOU2 & OUND & NHOK & NDHO & IVI & $\pm \mathbf{E T}$ \\
\hline 1 & Senna alata & $0,00 \mathrm{a}$ & $175,00 \mathrm{a}$ & $0,00 \mathrm{a}$ & $24,56 a$ & $4,85 \mathrm{a}$ & 204,42 & 75,65 \\
\hline 2 & Voacanga spp. & $57,84 \mathrm{a}$ & $0,00 \mathrm{a}$ & $6,66 \mathrm{a}$ & $25,96 a$ & $41,99 \mathrm{a}$ & 132,46 & 24,07 \\
\hline 4 & Milicia excelsa & $0,00 \mathrm{a}$ & $25,00 \mathrm{a}$ & $29,17 \mathrm{a}$ & $5,96 a$ & $14,45 \mathrm{a}$ & 74,58 & 12,33 \\
\hline 5 & Albizia adianthifolia & $0,00 \mathrm{a}$ & $25,00 \mathrm{a}$ & $15,31 \mathrm{a}$ & $9,30 \mathrm{a}$ & $11,78 \mathrm{a}$ & 61,39 & 9,10 \\
\hline 6 & Ricinodendron heudelotii & $20,10 a$ & $0,00 \mathrm{a}$ & $11,35 \mathrm{a}$ & $15,26 \mathrm{a}$ & $14,41 \mathrm{a}$ & 61,12 & 7,52 \\
\hline 8 & Ficus exasperata & $10,05 \mathrm{a}$ & $0,00 \mathrm{a}$ & $24,67 \mathrm{a}$ & $15,26 \mathrm{a}$ & $8,26 a$ & 58,25 & 9,12 \\
\hline 9 & Albizia zygia & $0,00 \mathrm{a}$ & $0,00 \mathrm{a}$ & $6,66 \mathrm{a}$ & $11,93 a$ & $20,12 \mathrm{a}$ & 38,72 & 8,54 \\
\hline 10 & Lophira lanceolata & $0,00 \mathrm{a}$ & $0,00 \mathrm{a}$ & $10,62 \mathrm{a}$ & $24,56 \mathrm{a}$ & $0,00 \mathrm{a}$ & 35,19 & 10,82 \\
\hline 11 & Terminalia mantalis & $20,10 \mathrm{a}$ & $0,00 \mathrm{a}$ & $4,68 \mathrm{a}$ & $5,96 a$ & $0,00 \mathrm{a}$ & 30,75 & 8,25 \\
\hline 12 & T. laxiflora & $0,00 \mathrm{a}$ & $0,00 \mathrm{a}$ & $29,89 a$ & $0,00 \mathrm{a}$ & $0,00 \mathrm{a}$ & 29,89 & 13,37 \\
\hline 16 & Cylicodiscus gabonensis & $0,00 \mathrm{a}$ & $25,00 \mathrm{a}$ & $0,00 \mathrm{a}$ & $0,00 \mathrm{a}$ & $1,37 \mathrm{a}$ & 26,37 & 11,04 \\
\hline 17 & Azadirachta indica & $25,98 \mathrm{a}$ & $0,00 \mathrm{a}$ & $0,00 \mathrm{a}$ & $0,00 \mathrm{a}$ & $0,00 \mathrm{a}$ & 25,98 & 11,62 \\
\hline 18 & Trichilia rubescens & $0,00 \mathrm{a}$ & $25,00 \mathrm{a}$ & $0,00 \mathrm{a}$ & $0,00 \mathrm{a}$ & $0,00 \mathrm{a}$ & 25,00 & 11,18 \\
\hline 19 & Eribroma oblongum & $21,81 \mathrm{a}$ & $0,00 \mathrm{a}$ & $0,00 \mathrm{a}$ & $0,00 \mathrm{a}$ & $2,08 \mathrm{a}$ & 23,89 & 9,57 \\
\hline 20 & Pterygota macrocarpa & $20,10 a$ & $0,00 \mathrm{a}$ & $0,00 \mathrm{a}$ & $0,00 \mathrm{a}$ & $1,37 \mathrm{a}$ & 21,47 & 8,85 \\
\hline 21 & Croton olingadrum & $10,05 \mathrm{a}$ & $0,00 \mathrm{a}$ & $4,68 \mathrm{a}$ & $5,96 \mathrm{a}$ & $0,00 \mathrm{a}$ & 20,70 & 4,27 \\
\hline 22 & Bridelia ferruginea & $10,05 \mathrm{a}$ & $0,00 \mathrm{a}$ & $0,00 \mathrm{a}$ & $9,30 \mathrm{a}$ & $0,00 \mathrm{a}$ & 19,35 & 5,31 \\
\hline 23 & Dracaena arborea & $10,05 \mathrm{a}$ & $0,00 \mathrm{a}$ & $6,66 \mathrm{a}$ & $0,00 \mathrm{a}$ & $1,37 \mathrm{a}$ & 18,08 & 4,52 \\
\hline & Probabilité & 0,016 & 0,002 & $\mathbf{0 , 0 2 3}$ & 0,010 & 0,176 & \multicolumn{2}{|c|}{$0,0001 * * *$} \\
\hline
\end{tabular}

L'effectif global à Ndikiniméki est constitué de 72 espèces reparties en 63 genres et 29 familles. Cette végétation d'ombrage est composée d'arbres utiles pour leur bois (Terminalia superba, Milicia excelsa, Triplochiton scleroxylon, Piptadeniastrum africanum, Entandrophragma spp.) ou pour leur graines condimentaires (Tetrapleura tetraptera, Ricinodendron heudelotii) ou oléo-protéagineuses (Irvingia gabonensis, Irvingia grandifolia) (Zapfack et al., 2000; Mapongmetsem et al., 2016; Dong et al., 2017).

Parmi les familles écologiquement importantes, les Moraceae occupent la première position avec un FIV de 26,96\%. Elles sont suivies par 
celles des Apocynaceae (25,66\%), Meliaceae (24,95\%), des Caesalpiniaceae et Euphorbiaceae avec chacun $24,11 \%$ (Tableau 7). L'analyse de variance montre une différence significative entre les indices d'importance écologique des familles $(\mathrm{P}=0,001<0,05)$ pour les localités Oundjock et Ndokohok. Alors que cette variation est apparente pour les localités Bognombang et Etoundou $2(\mathrm{P}>0,05)$. Par leur densité relative élevée, certaines espèces appartenant à ces familles impriment à la mosaïque forêt-savane de Ndikiniméki une physionomie originale. Ces familles sont majoritairement constituées d'espèces appartenant au front pionnier de reconquête forestière sur les savanes. Les espèces qui caractérisent ces familles sont entre autres Voacanga spp. (Apocynaceae), Milicia excelsa (Moraceae), Albizia zygia et Albizia adianthifolia (Mimosaceae), Ricinodendron heudelotii (Euphorbiaceae), Ficus exasperata (Moraceae) et Psychotria sp. (Rubiaceae).

Tableau 7 : Family Importance Value (FIV) des espèces locales dans l'Arrondissements de Ndikiniméki.

\begin{tabular}{c|ccccc|cc}
\hline Familles & BOGN & ETOU2 & OUND & NHOK & NDHO & FIV & $\mathbf{\pm}$ ET \\
\hline Moraceae & $1 \mathrm{a}$ & $2 \mathrm{a}$ & $19 \mathrm{ab}$ & $4 \mathrm{a}$ & $41 \mathrm{~b}$ & 26,96 & 17,07 \\
Apocynaceae & $7 \mathrm{a}$ & $0 \mathrm{a}$ & $2 \mathrm{ab}$ & $9 \mathrm{a}$ & $63 \mathrm{~b}$ & 25,66 & 26,41 \\
Meliaceae & $3 \mathrm{a}$ & $1 \mathrm{a}$ & $0 \mathrm{ab}$ & $0 \mathrm{a}$ & $13 \mathrm{~b}$ & 24,95 & 5,50 \\
Caesalpiniaceae & $0 \mathrm{a}$ & $11 \mathrm{a}$ & $2 \mathrm{ab}$ & $10 \mathrm{a}$ & $19 \mathrm{~b}$ & 24,11 & 7,64 \\
Euphorbiaceae & $9 \mathrm{a}$ & $0 \mathrm{a}$ & $4 \mathrm{ab}$ & $7 \mathrm{a}$ & $18 \mathrm{~b}$ & 24,11 & 6,73 \\
Mimosaceae & $1 \mathrm{a}$ & $2 \mathrm{a}$ & $7 \mathrm{ab}$ & $4 \mathrm{a}$ & $38 \mathrm{~b}$ & 21,32 & 15,60 \\
Combretaceae & $2 \mathrm{a}$ & $0 \mathrm{a}$ & $37 \mathrm{ab}$ & $7 \mathrm{a}$ & $5 \mathrm{~b}$ & 19,02 & 15,22 \\
Bignoniaceae & $1 \mathrm{a}$ & $0 \mathrm{a}$ & $1 \mathrm{ab}$ & $0 \mathrm{a}$ & $11 \mathrm{~b}$ & 16,27 & 4,72 \\
Sterculiaceae & $9 \mathrm{a}$ & $2 \mathrm{a}$ & $4 \mathrm{ab}$ & $4 \mathrm{a}$ & $59 \mathrm{~b}$ & 14,78 & 24,40 \\
Myrtaceae & $8 \mathrm{a}$ & $2 \mathrm{a}$ & $4 \mathrm{ab}$ & $11 \mathrm{a}$ & $44 \mathrm{~b}$ & 12,58 & 17,24 \\
Anacardiaceae & $35 \mathrm{a}$ & $23 \mathrm{a}$ & $44 \mathrm{ab}$ & $51 \mathrm{a}$ & $121 \mathrm{~b}$ & 11,99 & 38,46 \\
Rutaceae & $14 \mathrm{a}$ & $8 \mathrm{a}$ & $116 \mathrm{ab}$ & $64 \mathrm{a}$ & $106 \mathrm{~b}$ & 9,79 & 50,19 \\
Caricaceae & $12 \mathrm{a}$ & $15 \mathrm{a}$ & $53 \mathrm{ab}$ & $27 \mathrm{a}$ & $90 \mathrm{~b}$ & 8,62 & 32,58 \\
Rubiaceae & $2 \mathrm{a}$ & $0 \mathrm{a}$ & $2 \mathrm{ab}$ & $0 \mathrm{a}$ & $4 \mathrm{~b}$ & 7,36 & 1,67 \\
Agavaceae & $1 \mathrm{a}$ & $0 \mathrm{a}$ & $2 \mathrm{ab}$ & $0 \mathrm{a}$ & $1 \mathrm{~b}$ & 6,71 & 0,84 \\
Verbenaceae & $0 \mathrm{a}$ & $0 \mathrm{a}$ & $4 \mathrm{ab}$ & $8 \mathrm{a}$ & $6 \mathrm{bb}$ & 6,29 & 3,58 \\
Ulmaceae & $1 \mathrm{a}$ & $0 \mathrm{a}$ & $0 \mathrm{ab}$ & $0 \mathrm{a}$ & $0 \mathrm{~b}$ & 5,38 & 0,45 \\
Clusiaceaea & $0 \mathrm{a}$ & $0 \mathrm{a}$ & $0 \mathrm{ab}$ & $0 \mathrm{a}$ & $17 \mathrm{~b}$ & 4,84 & 7,60 \\
Bombacaceae & $1 \mathrm{a}$ & $0 \mathrm{a}$ & $3 \mathrm{ab}$ & $2 \mathrm{a}$ & $7 \mathrm{~b}$ & 4,24 & 2,70 \\
Araliaceae & $0 \mathrm{a}$ & $0 \mathrm{a}$ & $0 \mathrm{ab}$ & $0 \mathrm{a}$ & $12 \mathrm{~b}$ & 4,17 & 5,37 \\
Ochnaceae & $0 \mathrm{a}$ & $0 \mathrm{a}$ & $4 \mathrm{ab}$ & $5 \mathrm{a}$ & $0 \mathrm{~b}$ & 4,11 & 2,49 \\
Loganiaceae & $0 \mathrm{a}$ & $0 \mathrm{a}$ & $0 \mathrm{ab}$ & $1 \mathrm{a}$ & $6 \mathrm{~b}$ & 3,75 & 2,61 \\
Lauraceae & $14 \mathrm{a}$ & $2 \mathrm{a}$ & $49 \mathrm{ab}$ & $12 \mathrm{a}$ & $8 \mathrm{~b}$ & 3,72 & 18,47 \\
Boraginaceae & $0 \mathrm{a}$ & $0 \mathrm{a}$ & $0 \mathrm{ab}$ & $0 \mathrm{a}$ & $5 \mathrm{~b}$ & 3,53 & 2,24 \\
Fabaceae & $0 \mathrm{a}$ & $0 \mathrm{a}$ & $0 \mathrm{ab}$ & $0 \mathrm{a}$ & $2 \mathrm{~b}$ & 3,17 & 0,89 \\
\hline Probabilité & $\mathbf{0 , 1 8 8}$ & $\mathbf{0 , 3 2 8}$ & $\mathbf{0 , 0 0 6}$ & $\mathbf{0 , 0 3 1}$ & $\mathbf{0 , 0 0 0 1}$ & $\mathbf{0 , 0 0 0 1}$ & $* * *$ \\
\hline & & & & & & &
\end{tabular}


Du point de vue densité des espèces, la famille des Apocynaceae vient au premier rang suivi des Moraceae, Mimosaceae, Combretaceae, Caesalpiniaceae, Euphorbiaceae, Verbenaceae, Clusiaceae et Malvaceae (Fig. 1A). Les Bignoniaceae, Meliaceae, Araliaceae, Ochnaceae et Rubiaceae sont faiblement représentées. Les agroforêts de Ndokononoho sont celles qui présentent à un intervalle de confiance de $95 \%$, l'indice de raréfaction le plus élevé $(68,84 \%)$. Ces agroforêts comptent un grand nombre d'espèces et de familles rares. Elles sont suivies des agroforêts des localités de Oundjock (38,83\%), Ndokohok (37,71\%), Bognombang (33,37\%) et enfin d'Etoundou $2(19,63 \%)$ (Fig. 1B).
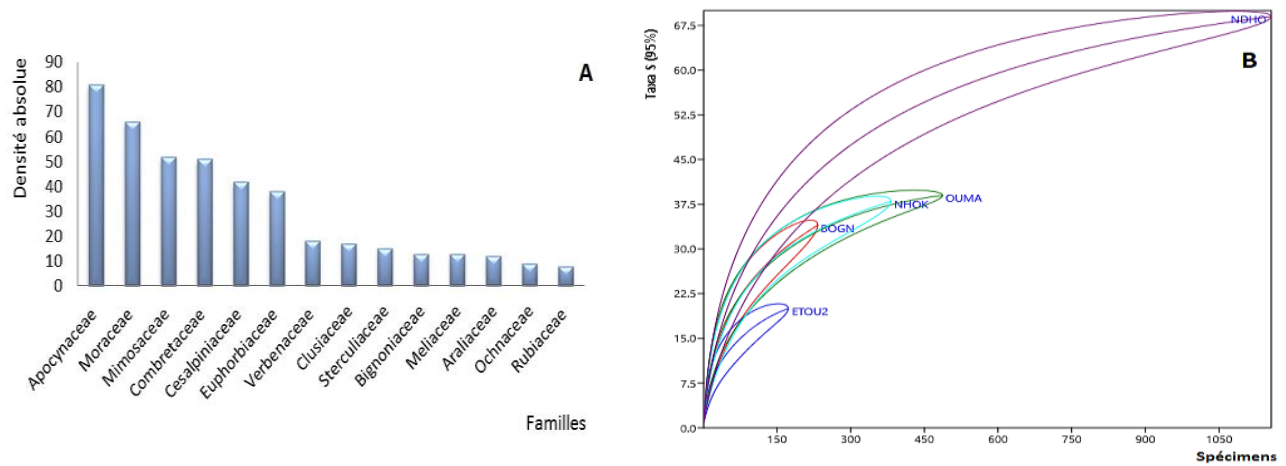

Figure 2 : Ecologie des familles à Ndikiniméki : Densité spécifique par famille (A) ; Indice de raréfaction par famille (B).

La famille des Euphorbiaceae est la plus diversifiée avec sept espèces, suivie des Caesalpiniaceae (six), des Moraceae, Apocynaceae, Mimosaceae et Malvaceae avec chacune cinq espèces. Les valeurs des différents paramètres écologiques (Fréquence, FIV, IVI) traduisent l'importance agro-écologique de la composante ligneuse agroforestière dans la dynamique de la végétation de la mosaïque forêt-savane de Ndikiniméki.

\section{Diversité écologique dans les agroforêts}

Les agroforêts de Ndokononoho et Oundjock ont l'indice de Shannon le plus élevé avec respectivement 3,06 et 2,97. Ce résultat traduit une plus grande diversité de leurs agroforêts. Cette diversité est aussi importante dans les agroforêts des localités de Ndokohok $(2,80)$ et Bognombang $(2,79)$ (Tableau 8). Les agroforêts les moins diversifiées sont celles de la localité d'Etoundou $2(2,23)$. Les localités de Oundjock $(0,81)$, Bognombang $(0,79)$ et Ndokohok $(0,77)$ sont celles dont les agroforêts présentent les indices d'équitabilité de Piélou les plus élevés. Cette tendance traduit une faible importance d'espèces dominantes dans ces agroforêts. Par contre la faible équitabilité de Piélou des agroforêts de Ndokononoho $(0,72)$ traduit une plus grande importance d'espèces dominantes. L'analyse de variance ne montre 
pas de différence significative entre ces divers indices de diversité dans ces différentes agroforêts $(\mathrm{P}>0,05)$.

Tableau 8 : Indices de diversité écologiques dans les différentes agroforêts de Ndikiniméki.

\begin{tabular}{l|lllll|lc}
\hline Localités & BOGN & ETOU2 & OUND & NHOK & NDHO & Moyenne \pm ET & Probabilité \\
\hline Fréquence_S & 34,00 & 20,00 & 39,00 & 38,00 & 69,00 & $40 \pm 17,9$ & $\mathbf{0 , 5 2 6}$ \\
Effectif_E & 233,00 & 173,00 & 487,00 & 382,00 & 1156,00 & $486,2 \pm 394,2$ & $\mathbf{0 , 0 0 0 1}$ \\
Dominance_D & 0,10 & 0,16 & 0,07 & 0,10 & 0,09 & $0,10 \pm 0,03$ & $\mathbf{0 , 9 9 9}$ \\
Simpson_1-D & 0,90 & 0,84 & 0,93 & 0,90 & 0,91 & $0,89 \pm 0,03$ & $\mathbf{0 , 9 8 9}$ \\
Shannon_H & 2,79 & 2,23 & 2,97 & 2,80 & 3,06 & $2,77 \pm 0,32$ & $\mathbf{0 , 9 6 5}$ \\
Evenness_e^H/S & 0,48 & 0,47 & 0,50 & 0,43 & 0,31 & $0,43 \pm 0,08$ & $\mathbf{0 , 9 9 4}$ \\
Equitability_J & 0,79 & 0,75 & 0,81 & 0,77 & 0,72 & $0,76 \pm 0,04$ & $\mathbf{0 , 9 9 0}$ \\
Fisher_alpha & 10,96 & 5,85 & 9,98 & 10,49 & 16,09 & $10,67 \pm 3,65$ & $\mathbf{0 , 8 6 5}$ \\
\hline
\end{tabular}

Ces indices de diversité confirment l'importance écologique des espèces Elaeis guineensis (47\%), Dacryodes edulis (37\%), Mangifera indica (31\%), Carica papaya (29\%), Citrus sinensis (20\%), Citrus reticulate $(17 \%)$, Persea americana (16\%), Voacanga spp (14\%) et Psidium guajava (14\%).

Comme autre perception, les populations locales ont remarqué la disparition, la diminution et la prolifération de certaines espèces végétales dans la zone. Ils soulignent l'invasion des adventices qui causent de sérieux problèmes aux cultures. L'écologie de ces espèces végétales montre qu'elles peuvent se développer correctement sous un climat tropical caractérisé par une péjoration pluviométrique (Akobundu et Agyakwa, 1989). Même constat pour Chromolaena odorata (Asteraceae), Digitaria spp (Poaceae), Pennisetum purpureum (Poaceae), Imperata cylindrica (Poaceae), Costus afer (Costaceae) et Ageratum sp. (Asteraceae) (Achoundong et al., 1996b) qui prolifèrent aisément dans les parcelles en zone d'écotone forêt-savane. Les espèces comme Pterygota macrocarpa, Garcinia cola, Cylicodiscus gabonensis, Eribroma oblongum, Diospyros sp et Nesogordonia papaverifera sont de plus en plus rares dans les agroforêts de la zone. Il faut aller de plus en plus loin dans les forêts pour les rencontrer, preuve qu'elles sont en voie de disparition.

\section{Biomasse épigée et stock de carbone}

Les espèces Mangifera indica (64,80 t/ha), Dacryodes edulis $(41,23$ t/ha), Spondias cytherea $(37,41 \mathrm{t} / \mathrm{ha})$, Ricinodendron heudelotii $(24,81 \mathrm{t} / \mathrm{ha})$, Albizia zygia (14,86 t/ha) et Ficus exasperata (13,65 t/ha) sont celles qui séquestrent le plus de carbone dans les agroforêts de la zone (Tableau 9). Globalement l'analyse de variance ne révèle pas une différence significative entre les biomasses et stock de carbone dans les agroforêts de ces différentes localités $(\mathrm{P}=0,517>0,05)$, cependant une différence très significative existe entre les biomasses et stock de carbone des agroforêts de Ndokononoho (a) et celles des 4 autres localités (b). 
A Etoundou 2, ce sont Mangifera indica, Dacryodes edulis et Cola acuminata qui sont les plus fréquentes, mais les espèces qui stockent le plus sont respectivement : Mangifera indica (118,8 t), Dacryodes edulis $(84,1 \mathrm{t})$ et Spondias cytherea (59,8 t). Dans la localité de Oundjock, ce sont : Ceiba pentandra (98,2 t), Ficus exasperata (77,8 t) et Dacryodes edulis $(59,3$ t) qui sont respectivement celles qui stockent le plus. Du côté de Ndokohok, ce sont respectivement Mangifera indica (207,6 t), Vitex doniana (121,6 t) et Delonix regia $(69,5$ t). Du côté de Ndokononoho par contre, ce sont Mangifera indica (301,1 t), Dacryodes edulis (295,1 t) et Ricinodendron heudelotii (291,1 t).

Tableau 9 : Biomasse épigée totale $\mathbf{Y}(\mathrm{t}$ et $\mathrm{t} / \mathrm{ha}$ ) et quantité totale de carbone accumulées Qc (t/ha) par 24 essences des agroforêts.

\begin{tabular}{|c|c|c|c|c|c|c|c|c|}
\hline Espèces & BOGN & ETOU2 & OUND & NHOK & NDHO & To Y $(t) \pm$ ET & $\begin{array}{l}\text { To Y } \\
\text { (t/ha) }\end{array}$ & $\begin{array}{l}\text { To Qc } \\
\text { (t/ha) }\end{array}$ \\
\hline Mangifera indica & $199,7 b$ & $118,8 b$ & $57,9 \mathrm{~b}$ & $207,6 b$ & $301,1 \mathrm{a}$ & $885,1 \pm 92,77$ & 64,8 & 30,45 \\
\hline Dacryodes edulis & $61,1 \mathrm{~b}$ & $84,1 \mathrm{~b}$ & $59,3 b$ & $63,6 b$ & $295,1 \mathrm{a}$ & $563,2 \pm 102,48$ & 41,23 & 19,38 \\
\hline Spondias cytherea & $99,9 b$ & $59,8 b$ & $53,8 b$ & $41,7 b$ & $255,7 \mathrm{a}$ & $511 \pm 88,56$ & 37,41 & 17,58 \\
\hline $\begin{array}{l}\text { Ricinodendron } \\
\text { heudelotii }\end{array}$ & $17 \mathrm{~b}$ & $0 \mathrm{~b}$ & $30,8 b$ & $\mathrm{Ob}$ & $291,1 \mathrm{a}$ & $338,9 \pm 125,51$ & 24,81 & 11,66 \\
\hline Albizia zygia & $\mathrm{Ob}$ & $0 \mathrm{~b}$ & $\mathrm{Ob}$ & $22,3 b$ & $180,8 \mathrm{a}$ & $203,1 \pm 78,96$ & 14,87 & 6,99 \\
\hline Ficus exasperata & $0 \mathrm{~b}$ & $0 \mathrm{~b}$ & $77,8 b$ & Ob & $108,7 \mathrm{a}$ & $186,5 \pm 52,23$ & 13,65 & 6,42 \\
\hline Milicia excelsa & $0 \mathrm{~b}$ & $0 b$ & Ob & $0 \mathrm{~b}$ & $184,3 \mathrm{a}$ & $184,3 \pm 82,42$ & 13,49 & 6,34 \\
\hline Cola acuminata & $2 b$ & $20,6 b$ & $0 \mathrm{~b}$ & Ob & $135,3 \mathrm{a}$ & $157,8 \pm 58,63$ & 11,55 & 5,43 \\
\hline Vitex doniana & $0 \mathrm{~b}$ & $0 \mathrm{~b}$ & $0 \mathrm{~b}$ & $121,6 b$ & $0 \mathrm{a}$ & $121,6 \pm 54,38$ & 8,9 & 4,18 \\
\hline Afzelia bipindensis & Ob & $\mathrm{Ob}$ & Ob & Ob & $121 \mathrm{a}$ & $121 \pm 54,11$ & 8,86 & 4,16 \\
\hline Persea americana & $21,7 \mathrm{~b}$ & $2,4 b$ & $45,1 \mathrm{~b}$ & $26,7 b$ & $6,7 \mathrm{a}$ & $102,6 \pm 17,05$ & 7,51 & 3,53 \\
\hline Ceiba pentandra & ob & $0 \mathrm{~b}$ & $98,2 b$ & ob & $0 \mathrm{a}$ & $98,2 \pm 43,92$ & 7,19 & 3,38 \\
\hline Pterygota macrocarpa & $71,4 b$ & $0 \mathrm{~b}$ & ob & $0 \mathrm{~b}$ & $16,5 \mathrm{a}$ & $87,9 \pm 30,92$ & 6,44 & 3,02 \\
\hline Musanga cecropioides & Ob & $0 b$ & $0 \mathrm{~b}$ & $0 b$ & $85 \mathrm{aa}$ & $85 \pm 38,01$ & 6,23 & 2,93 \\
\hline Khaya ivorensis & $0 \mathrm{~b}$ & $0 \mathrm{~b}$ & $0 \mathrm{~b}$ & $0 \mathrm{~b}$ & $77 \mathrm{a}$ & $77 \pm 34,44$ & 5,64 & 2,65 \\
\hline Garcinia cola & $0 \mathrm{~b}$ & $0 \mathrm{~b}$ & $0 \mathrm{~b}$ & $0 \mathrm{~b}$ & $69,7 \mathrm{a}$ & $69,7 \pm 31,17$ & 5,1 & 2,4 \\
\hline Delonix regia & $0 \mathrm{~b}$ & $\mathrm{Ob}$ & $0 \mathrm{~b}$ & $69,5 b$ & 0aa & $69,5 \pm 31,08$ & 5,09 & 2,39 \\
\hline Hallea stipulosa & $66,1 b$ & $0 \mathrm{~b}$ & Ob & Ob & $0 \mathrm{a}$ & $66,1 \pm 29,56$ & 4,84 & 2,27 \\
\hline Terminalia superba & Ob & $0 b$ & $59,3 b$ & $0 \mathrm{~b}$ & $0 \mathrm{a}$ & $59,3 \pm 26,52$ & 4,34 & 2,04 \\
\hline Gambeya beguei & $\mathrm{Ob}$ & $0 b$ & Ob & $0 b$ & $40,4 a$ & $53,8 \pm 18,07$ & 3,93 & 1,85 \\
\hline Polyscia fulva & $0 \mathrm{~b}$ & $0 \mathrm{~b}$ & $0 \mathrm{~b}$ & $0 \mathrm{~b}$ & $43,4 \mathrm{a}$ & $52,8 \pm 19,41$ & 3,86 & 1,82 \\
\hline Lovoa trichilioides & $0 \mathrm{~b}$ & $0 \mathrm{~b}$ & $0 \mathrm{~b}$ & $0 \mathrm{~b}$ & $44,4 \mathrm{a}$ & $51,8 \pm 19,86$ & 3,79 & 1,78 \\
\hline Fagara heitzii & $0 \mathrm{bb}$ & $0 \mathrm{~b}$ & $0 \mathrm{~b}$ & $0 \mathrm{~b}$ & $51,1 \mathrm{a}$ & $51,1 \pm 22,85$ & 3,74 & 1,76 \\
\hline $\begin{array}{l}\text { Cylicodiscus } \\
\text { gabonensis }\end{array}$ & $\mathrm{Ob}$ & $34,8 b$ & $0 \mathrm{~b}$ & $0 \mathrm{~b}$ & $16 \mathrm{a}$ & $50,8 \pm 15,42$ & 3,72 & 1,75 \\
\hline Total (t) & 538,9 & 320,5 & 482,2 & 553 & 2323,3 & $4248,1 \pm 1168,32$ & 310,99 & 146,16 \\
\hline Probabilité & 0,946 & 0,642 & 0,080 & 0,919 & 0,0001 & $\mathbf{0 , 5 1 7}$ & & \\
\hline
\end{tabular}

Dans l'ensemble de la zone concernant les Arecaceae, la biomasse totale est de $0,99 \mathrm{t} / \mathrm{ha}$, soit une quantité totale de carbone séquestrée de 0,46 tC/ha (Tableau 10). Les espèces concernées sont entre autres ; Elaeis 
guineensis, Borassus aethiopum et Coco nucifera. L'analyse statistique ne montre pas de différence significative $(\mathrm{P}>0,05)$ entre les différentes agroforêts de la zone. Il faut noter une nette prédominance d'Elaeis guineensis $(0,72 \mathrm{t} / \mathrm{ha})$ par ailleurs seule espèce commune à toutes les localités inspectées.

Tableau 10 : Biomasses aériennes et quantité de carbone séquestré par les Arecaceae dans les agroforêts.

\begin{tabular}{cc|cccccc|cc}
\hline \multirow{2}{*}{ Villages } & Espèces & \multicolumn{2}{|c}{ Elaeis guineensis } & \multicolumn{2}{c}{ Cocos nucifera } & \multicolumn{2}{c}{ Borassus aethiopum } & \multicolumn{2}{c}{ Total (t) } \\
\cline { 2 - 10 } & Quantité & Biomasse & $\begin{array}{c}\text { Stock de } \\
\text { Carbone }\end{array}$ & Biomasse & $\begin{array}{c}\text { Stock de } \\
\text { Carbone }\end{array}$ & Biomasse & $\begin{array}{c}\text { Stock de } \\
\text { Carbone }\end{array}$ & Biomasse & $\begin{array}{c}\text { Stock de } \\
\text { Carbone }\end{array}$ \\
\hline Bognombang & 1,4 & 0,66 & 0,37 & 0,17 & 0 & 0 & 1,77 & 0,83 \\
Etoundou 2 & 1,52 & 0,71 & 0,21 & 0,1 & 0 & 0 & 1,73 & 0,81 \\
Oundjock & 0,21 & 0,1 & 0,54 & 0,25 & 0 & 0 & 0,75 & 0,35 \\
Ndokohok & 1,85 & 0,87 & 1,1 & 0,52 & 0,47 & 0,22 & 3,42 & 1,61 \\
Ndokononoho & 4,87 & 2,29 & 1,08 & 0,51 & 0 & 0 & 5,95 & 2,8 \\
\hline Total (t) & $\mathbf{9 , 8 5} \pm \mathbf{1 , 7 3}$ & $\mathbf{4 , 6 3 \pm 0 , 8 1}$ & $\mathbf{3 , 3} \pm \mathbf{0 , 4 1}$ & $\mathbf{1 , 5 5} \pm \mathbf{0 , 1 9}$ & $\mathbf{0 , 4 7 \pm 0 , 2 1}$ & $\mathbf{0 , 2 2} \pm \mathbf{0 , 0 9}$ & $\mathbf{1 3 , 6 2 \pm 4 , 8 0}$ & $\mathbf{6 , 4} \pm \mathbf{2 , 2 6}$ \\
Total (t/ha) & $\mathbf{0 , 7 2}$ & $\mathbf{0 , 3 3}$ & $\mathbf{0 , 2 3}$ & $\mathbf{0 , 1 1}$ & $\mathbf{0 , 0 3}$ & $\mathbf{0 , 0 2}$ & $\mathbf{0 , 9 9}$ & $\mathbf{0 , 4 6}$ \\
\hline
\end{tabular}

Nous avons une absence remarquée de Borassus aethiopum dans les agroforêts de Bognombang, Etoundou 2, Oundjock et Ndokononoho. Les agroforêts de Oundjock ont la plus faible biomasse de Elaeis guineensis $(0,21$ t/ha) tans dis que celles de Ndokohok ont la biomasse la plus élevée de Coco nucifera $(1,1 \mathrm{t} / \mathrm{ha})$, suivie de celles de Ndokononoho (1,08 t/ha).

Globalement dans la zone, l'on obtient une biomasse ligneuse (hormis les Arecaceae) de 4248,1t (310,99t/ha) pour un stock de carbone de 1996,60t $(146,16 \mathrm{t} / \mathrm{ha})$ ce qui équivaut à un stock de $\mathrm{CO}_{2}$ de $7327,54 \mathrm{t} \mathrm{CO}_{2}$. Les ligneux de la famille des Arecaceae donne une biomasse de 13,62t $(0,99 \mathrm{t} / \mathrm{ha})$ pour un stock de carbone de 6,40t (0,46t/ha) soit un stock de $\mathrm{CO}_{2}$ de $23,49 \mathrm{tCO}_{2}$. Ce qui nous donne une biomasse ligneuse totale dans la zone de 4261,72t (311,98t/ha) pour un stock de carbone total de 2003t (146,62t/ha) pour un total de stock de $\mathrm{CO}_{2}$ de $7351,03 \mathrm{CO}_{2}$. Si le crédit carbone venait à être payé à 10

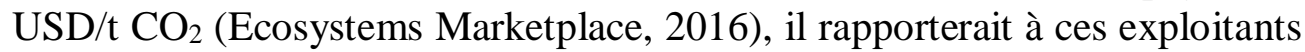
un total de 73510,03 USD, soit 44.026.788,87 Franc CFA ( 1 USD = 598,92 Franc CFA).

\section{Discussion}

Concernant les caractéristiques biophysiques et fonctionnelles des agroforêts, les jeunes agroforêts (moins de 15 ans) sont les plus importantes dans la zone $(31,42 \%)$ suivie de près par celles dont l'âge est supérieur à 45 ans $(31,08 \%)$. Ces 4 tranches d'âges d'agroforêts varient significativement. Une telle situation serait due dans la plupart des cas à l'acquisition de ces parcelles par héritage ou par achat, et ou les vieilles agroforêts héritées sont en train d'être remplacées par l'établissement de nouvelles. Ce constat s'explique par l'arrivée de nouveaux retraités dans la zone qui s'établissent de nouvelles agroforêts. Ces résultats sont contraires à ceux obtenus dans les 
Hautes terres de l'Adamaoua (Hamawa, 2005 ; Mamah, 2007) et de Dong (2010) et Mapongmetsem et al. (2016) à Bafia. On remarque également que plus ces villages sont éloignés du centre urbain de Bafia, plus leurs agroforêts sont âgées et composées d'un effectif élevé de grands arbres locaux. Il convient de relever toutefois que certains exploitants n'arrivent pas à donner avec exactitude l'âge de leur agroforêt.

La classe de superficie supérieure à $1500 \mathrm{~m}^{2}$ vient au premier rang avec $32 \%$ suivie de la classe inférieure à $500 \mathrm{~m}^{2}$ avec $25,9 \%$. Ces taux s'expliquent par le pouvoir d'achat et l'enthousiasme des paysans à posséder des parcelles plus ou moins grandes. Nos résultats sont en désaccord avec ceux de Mapongmetsem et al. (2000) et Salbai (2001) qui ont travaillé sur la même problématique dans les hautes savanes guinéennes du Cameroun. Pour ces auteurs les jardins de case sont généralement de petites exploitations familiales, peu stratifiées et diversifiées comparés aux agroforêts types des zones forestières et péri-forestières qui sont plus étendues, étagées et complexes. Concernant les parcelles de superficie supérieures à $1500 \mathrm{~m}^{2}$, ce taux élevé s'explique par le fait que les paysans majoritairement pauvres et âgés, ne sont plus capables de gérer et d'entretenir de vastes étendues. Pour cela, ils créent une seule exploitation et adhèrent aux Groupes d'Initiative Communautaires (GIC) qui fonctionnent suivant un planning précis de rotation de défrichage et d'aménagement des agroforêts appartenant aux différents adhérents. Il est important de relever le taux assez élevé $(17,26 \%)$ des exploitants ne sachant pas avec exactitude la superficie ou les limites de leur exploitation. Cela pourrait être lié d'une part au faible taux de scolarisation de certains de ces exploitants, d'autre part au désintéressement quant aux procédures foncières et administratives qui apportent plus de précision sur l'identité de leurs propriétés, la plupart étant héritiers. De manière générale les grandes superficies agroforestières ne sont pas aussi communes dans la région du Centre et dans le Mbam et Inoubou en particulier comme le montre Dong (2010) et Mapongmetsem et al. (2016) dans leurs travaux réalisés à Bafia. Il faut par ailleurs noter qu'en zone forestière, les grandes superficies agroforestières sont des agroforêts à cacaoyers, à caféiers, à bananiers ou à palmiers à huile.

La production fréquente des tubercules (Manihot esculanta, Ipomoea batatas et Xanthosoma sagittifolium), de Légumineuses alimentaires (Phaseolus vulgaris) et de céréales (Zea mays) dans les agroforêts corroborent les résultats des travaux de Salbaï (2001) dans la région de Bélel puis à ceux de Dallière et Dounias (1998) dans la plaine Tikar du centre Cameroun. Les travaux de Mapongmetsem et al. (2016) dans la ville de Bafia le confirment également. Suivant l'ingéniosité du paysan, ces cultures peuvent être associées aux arbustes fruitiers divers (agrumes) et bananiers. Ces fruitiers sont plantés de préférence sur les côtés ou derrière la maison, à faible distance afin d'éviter 
les larcins (Tchatat, 1996 ; Mapongmetsem et al., 2000, 2009). Concernant les cultures de rente, la cacaoculture est la plus répandue avec une fréquence de $73 \%$ au vu de la relance du secteur. Ces résultats sont analogues à ceux de Jagoret et al. (2011, 2012) et de Jiofack et al. (2013) réalisés dans les agroforêts de diverses localités du Mbam et Inoubou dans le centre Cameroun. Ce résultat s'explique par le fait que la vente du cacao procure un important revenu aux ménages. Durant la décennie 80, la cacaoculture contribuait de 50 à $75 \%$ du budget total de $90 \%$ des ménages du Centre et du Sud Cameroun (Leplaideur, 1985). Ce constat a également été souligné chez les Tikar du Haut Mbam (Dounias, 1996b) et dans diverses localités du Centre et du Sud Cameroun (Oyono, 1999 ; Sonwa et al., 2000, 2002). Plusieurs auteurs au rang desquels, Kotto-Same et al., (1997), Gockowski et Sonwa (2011), Norgrove et hauser (2013) ont estimé les stocks de carbone des arbres associés dans les cacaoyères ombragées au Cameroun respectivement à $88,7 \mathrm{tC} / \mathrm{ha}, 107 \mathrm{tC} / \mathrm{ha}$ et $135,5 \mathrm{tC} / \mathrm{ha}$.

Comme stratégies développées par les paysans quant à leur perception sur les changements climatiques, ils optent pour l'abandon de certaines cultures ou variétés de culture, l'adoption de nouvelles cultures ou nouvelles variétés de culture, la rotation des cultures, la modification des emblavures, le changement d'itinéraire technique et même du régime alimentaire (Vissoh et al., 2004 ; Ogouwalé, 2004; Agossou, 2008). Des stratégies endogènes ont été également développées par les producteurs dans la gestion des sols de leur terroir : l'exploitation simultanée des différentes unités de paysage et le changement de sites de parcelles. Par ailleurs la consommation et la vente des cultures vivrières, fruitières et cultures de rente permettent de s'adapter au changement climatique, en ce sens les revenus obtenus permettent d'acheter les produits qui manquent et qui ne sont pas produits dans les agroforêts.

Concernant la composante ligneuse, Dacryodes edulis, Mangifera indica, les sub-ligneux bananiers fruits (Musa sapientum), bananiers plantains (Musa paradisiaca) et palmier à huile (Elaeis guineensis) sont les espèces les plus fréquentes dans les agroforêts de Ndikiniméki. Les résultats obtenus corroborent ceux de Jiofack et al. (2013) qui signalent que Mangifera indica vient au premier rang parmi les fruitiers exotiques rencontrés dans les agroforêts cacao de Bafia, mais atteste tout de même que le genre Citrus est également le plus diversifié de ces fruitiers avec quatre espèces. Ce résultat corrobore celui de Jagoret et al. (2012) dans les agroforêts à cacaoyers de Bokito et Mapongmetsem et al. (2016) dans les agroforêts de Bafia. L'oranger commun (Citrus sinensis) étant le plus abondant des quatre espèces et le plus apprécié à la consommation par les populations. Cette dominance du genre Citrus dans la strate arbustive des fruitiers exotiques est confirmée par Dong et al. (2017) dans les agroforêts de Makénéné. Ce fait s'explique par l'importance socio-économique, alimentaire et médicinale de ces Citrus ; que 
ce soit les fruits, les feuilles, les écorces et les racines. Pour Temgoua et al. (2019), ce sont les espèces Cola acuminata, Elaeis guineensis, Dacryodes edulis, Persea americana et Ficus exasperata qui sont les plus fréquentes et communes dans les systèmes agroforestiers des localités de Kwata, Bonkeng et Loum Cie dans l'Arrondissement de Loum, Département du Moungo. Senna alata (204,42\%), Voacanga spp. (132,46\%), Terminalia glaucescens $(84,25 \%)$, Milicia excelsa $(74,58 \%)$ et Albizia adianthifolia $(61,39 \%)$ sont les espèces qui présentent l'Importance Value Index (IVI) les plus élevés. Les Moraceae (26,96\%), Apocynaceae (25,66\%), Meliaceae (24,95\%), Caesalpiniaceae $(24,11 \%)$ et Euphorbiaceae $(24,11 \%)$ sont les familles écologiquement importantes dans ces agroforêts.

Cette importance écologique (IVI et FIV) traduit l'établissement dans cette zone de mosaïque forêt-savane via les agroforêts d'un peuplement arbustif dense qui s'établit en milieu ouvert comme un bouclier à l'avant des grands arbres de la forêt qui progressivement prennent place dans l'agroforêt. Cette végétation est composée en majorité d'arbustes puis de grands arbres qui ont moins de $20 \mathrm{~m}$ de hauteur comme le soulignait déjà Youta (1998). Ces espèces sont citées dans les travaux de Jiofack et al. (2013) et Jagoret et al. (2012) comme des arbres de couverture dans les agroforêts cacao de Bafia et Bokito. A ces arbres et arbustes s'ajoutent des lianes et buissons de la famille des Flacourtiaceae et Connaraceae. Dans cette zone l'on rencontre en premier dans sa partie nord des forêts semi-caducifoliées sur relief bullé d'érosion avec reliques d'éléments de forêts atlantiques à Caesalpiniaceae rares de type nordoccidental. Les localités Etoundou 2, Oundjock et Ndokohok sont concernés par ce type de végétation. En second se distingue la forêt atlantique à Caesalpiniaceae rares de type nord-occidental avec avancée d'éléments de forêts semi-caducifoliées. Dans cette catégorie, les Caesalpiniaceae grégaires sont absentes et les éléments atlantiques nombreux. On remarque aussi la présence de nombreuses espèces pionnières. Dans la zone d'étude, cette forêt couvre la partie centrale et méridionale à savoir les villages Bognombang et Ndokononoho.

Les agroforêts de Ndokononoho (3,06 bits), Oundjock (2,97 bits), Ndokohok (2,80 bits) et Bognombang (2,79 bits) ont l'indice de Shannon le plus élevé. Ce résultat traduit une plus grande diversité de leurs agroforêts, surtout en espèces d'importance socio-économique, des fruitiers exotiques et locaux à fruits charnus comestibles par l'homme ou par les animaux comme le soulignaient déjà Jagoret et al. (2012) et Jiofack et al. (2013). La baisse progressive de cet indice serait dû aux diverses activités d'urbanisation et à la proximité de ces agroforêts et localités du centre urbain de Ndikiniméki. Les divers autres indices renseignent sur le type floristique d'agroforêt (hétérogène ou homogène); et qui est constituée d'espèces aux exigences écologiques différentes (Warwick et al., 2002). Si la diversité est faible, alors les individus 
proches ont les mêmes types d'exigences, et l'agroforêt est dite homogène et est composée de types végétaux peu différents.

La variation de biomasses et des stocks de carbone s'explique d'une part par la densité assez élevée des espèces fruitières exotiques et grands arbres. D'autre part du fait des valeurs importantes de leur diamètre à hauteur de poitrine surtout chez les arbustes et arbres locaux comme le confirme les travaux de Mapongmetsem et al. (2016) et Dong et al. (2017) respectivement dans les agroforêts de la zone de Bafia et de Makénéné dans le Mbam et Inoubou. La biomasse aérienne et la quantité totale de carbone séquestré par les diverses espèces ligneuses montrent une corrélation avec le nombre d'individus recensés dans ces agroforêts. En d'autres termes, plus grand est le nombre d'individus inventoriés, plus élevée est la biomasse (Sonwa, 2004 ; Nair et al., 2009). Zapfack (2005) faisait une remarque semblable sur les types d'utilisation des terres de la région du Centre et du sud du Cameroun. Même constat chez les Arecaceae (Elaeis guineensis, Borassus aethiopum, Coco nucifera). Les résultats obtenus témoignent de la grande variabilité du nombre d'individus inventoriés, du type et du stade de croissance de l'individu (jeune pied ou pied adulte). Elaeis guineensis ou palmier à huile est l'espèce de la famille des Arecaceae qui stock le plus de carbone dans les agroforêts de Ndikiniméki. Ce résultat s'explique par la densité plus élevée de cette espèce dans les agroforêts, due à une plus grande préférence du palmier à huile par les paysans qui le trouve beaucoup plus rentable que Coco nucifera et Borassus aethiopum. La plupart de ces espèces et familles sont mentionnées dans les travaux de Albrecht et Kandji (2003); Montagnini et Nair (2004); Nair et al. (2009) comme offrant des opportunités de stockage et d'atténuation du changement climatique en synergie avec l'adaptation. Sonwa (2004) a estimé des stocks de carbone de 243 t/ha dans les cacaoyères au sud du Cameroun. Ces espèces contribuent aussi à d'autres services écologiques tels que la conservation (Saj et al., 2013, 2017). Le GIEC (2007) rapportait des superficies mondiales propices à l'agroforesterie allant de 585 à 1215 million d'hectare et présentant un potentiel technique d'atténuation de 1,1 à 2,2 Pétagramme de carbone d'ici environ 2050. Au vu de ces différents résultats sur la biomasse et le stock de carbone par la composante ligneuse des agroforêts de Ndikiniméki, il serait judicieux de les prendre en compte dans l'orientation et l'application des politiques du mécanisme REDD++ d'abord à l'échelle locale, nationale et voir sous-régionale.

\section{Conclusion}

Les agroforêts font partie du paysage agraire de l'Arrondissement de Ndikiniméki dans la région du centre Cameroun. Elles sont connues et désignées par diverses appellations par les populations locales de la zone, ce qui démontre l'importance de cette pratique dans la conservation de la 
phytodiversité et la sécurité alimentaire pour les populations. Les agroforêts jeunes (moins de 15 ans) et vieilles (supérieur à 45 ans) sont les plus importantes dans la zone. La classe de superficie supérieure à $1500 \mathrm{~m}^{2}$ occupe le premier rang $(32 \%)$ suivie de celle inférieure à $500 \mathrm{~m}^{2}(25,9 \%)$. Les cultures vivrières les plus fréquentes dans les agroforêts sont les tubercules (Manihot esculanta et Ipomoea batatas), les Légumineuse alimentaires (Phaseolus vulgaris et Arachis hypogaea) et le maïs (Zea mays). En effet, le maïs et le manioc constituent avec les gibiers la base de l'alimentation des populations de la zone. Il ressort de présentes investigations que la cacaoculture est la plus répandue des cultures de rente de la zone. Le safoutier (Dacryodes edulis) et le manguier (Mangifera indica) viennent au premier rang des fruitiers exotiques suivi des sub-ligneux (Musa spp.) et du palmier à huile (Elaeis guineensis). Des espèces locales, il ressort que Dacryodes edulis a la fréquence la plus élevée (84\%), suivie par les Sterculiaceae Cola spp. et Sterculia spp. Milicia excelsa vient en troisième rang, suivie de Myrianthus arboreus et Albizia zygia. L'effectif global à Ndikiniméki est constitué de 72 espèces reparties en 63 genres et 29 familles. Senna alata, Voacanga spp. et Terminalia glaucescens sont les espèces qui présentent l'Importance Value Index (IVI) les plus élevés. Les familles écologiquement importantes dans ces agroforêts sont entre autres les Moraceae, Apocynaceae et Meliaceae. Les agroforêts de Ndokononoho, Oundjock et Ndokohok sont les plus diversifiées en espèces fruitières et locales. Mangifera indica, Dacryodes edulis, Spondias cytherea et Ricinodendron heudelotii sont les espèces qui stockent le plus de carbone dans les agroforêts de la zone. Elaeis guineensis est l'espèce qui stocke le plus de carbone dans la famille des Arecaceae. La biomasse ligneuse totale dans la zone est estimée à 4261,72t pour un stock de carbone total de 2003t, ce qui correspond à un stock total de $\mathrm{CO}_{2}$ de $7351,03 \mathrm{t} \mathrm{CO}_{2}$ qui rapporterait aux exploitants un crédit carbone de 44.026.788,87 Franc CFA. Ces résultats attestent de l'efficacité de ces agroforêts dans la lutte contre le réchauffement climatique et pouvant servir dans l'orientation et l'application des politiques du mécanisme REDD++.

\section{Remerciements}

Les auteurs expriment leurs reconnaissances au Laboratoire de Biodiversité et Développement Durable de l'Université de Ngaoundéré et le Laboratoire de Botanique Appliquée de l'Université de Dschang pour leur réelle contribution à la réalisation de plusieurs investigations et activités scientifiques de ces travaux. Ils remercient également les experts anonymes pour leurs commentaires sur le manuscrit. 


\section{References:}

1. Achoundong G., Bonvallot J. et Youta Happi, 1996a. Contacts forêtsavane au Cameroun et Chromolaena odorata : considérations préliminaires. Symposium FAO : "3 rd International workshop on biological control and management of Chromolaena odorata". FAO, Abidjan, 15-19 Nov. 1993, AES-Univ. of Guam- ORSTOM-ICRAF, $202: 9$ p.

2. Achoundong G., Youta Happi et Bonvallot J., 1996b - Formation et évolution des recrûs sur savane. Symposium ECOFIT : "Dynamique à long terme des écosystèmes forestiers intertropicaux", ORSTOM/CNRS, Paris-Bondy, résumé : 5 p.

3. Agossou Sêsihouèdé M. D., 2008. Adaptation aux changements climatiques : perceptions, savoirs locaux et stratégies d'adaptation des producteurs des communes de Glazoué et de Savalou au centre du Bénin. Mémoire d'ingénieur agronome en économie, socioanthropologie et communication pour le développement rural, Faculté des sciences agronomiques de l'Université d'Abomey-Calavi, 173 p.

4. Akobundu, O. et Agyakwa C. W., 1989. Guide des adventices d'Afrique de l'ouest Institut International d'Agriculture Tropicale Ibadan, Nigéria p. 522

5. Albrecht A., et Kandji S., 2003. Carbon sequestration in tropical agroforestry Systems. Agriculture, Ecosystems and Environment. 99 : $12 \mathrm{p}$.

6. COMIFAC, 2010. Etat des forêts du bassin du Congo, 274 p.

7. Dagnelie P. 1998. Statistique théorique et appliquée. Tome 2. Bruxelles : De Boeck \& Larcier.

8. Dallière et Dounias, 1998. Agroforêts caféières et cacaoyères des Tikar (Cameroun central) -structures, dynamiques et alternatives de développement. Séminaire FORAFRI de Libreville - Session 3 : produits de la forêt ; $27 \mathrm{p}$.

9. De Baets, N., Lebel F., 2007. L’agroforesterie au Québec : Mémoire présenté à la commission pour l'avenir de l'agriculture et de l'agroalimentaire québécois, $165 \mathrm{p}$.

10. Dong E. A. B., 2010. Caracterisation biophysique des agroforêts dans la zone périurbaine de Bafia (Centre Cameroun). Mémoire de Master en Biologie des Organismes Végétaux. Université de Ngaoundéré, Cameroun, $68 \mathrm{p}$.

11. Dong Etchike A.B., Mapongmetsem P.M. \& Ngassoum M.B., 2017. Phytodiversité et stock de carbone dans les agroforêts du Mbam et Noubou au Cameroun. Cameroon Forum for Biological Sciences, 11(01), $13 \mathrm{p}$. 
12. Dounias, E., 1996a. Recrûs forestiers post-agricoles : perceptions et usages chez les Mvae du sud Cameroun forestier, Journal d'Agriculture Traditionnelle et de Botanique Appliquée, 38 (1).

13. Dounias, E., (Sous presse), 1996b. Ecotone forêt-savane et système agraire des Tikar du haut Mbam. In Dynamique à long terme des écosystèmes forestiers intertropicaux, M. Servant et S. ServantVildary (éds). Paris : ORSTOM-CNRS-UNESCO, 20 p.

14. Dounias, E., Hladik, A. et Hladik, C.M., 1996c. De la ressource disponible à la ressource exploitée : méthodes de quantification des ressources alimentaires dans les régions forestières et les savanes du Cameroun. In Froment, A., Garine, I.de, Binam Bikoï, Ch. et Loung, J.F. (eds). Anthropologie alimentaire et développement en Afri-que intertropicale : du biologique au social, (Paris : ORSTOML'Harmattan) ; 11 p.

15. Ecosystems Marketplace, 2016. State of the voluntary carbon market (p.25). USA: Ecosystems Marketplace, 58 p.

16. Fonds international de développement agricole (FIDA), 2008. Le FIDA et le changement climatique. Consultation sur la huitième reconstitution des ressources du FIDA. Rome : FIDA-IFAD, 27 p.

17. GIEC, 2002. Document technique V sur les changements climatiques et la biodiversité. Publié sous la direction de Habiba Gitay de l'Université nationale d'Australie Avelino Suárez du Ministère des Sciences, des Technologies et de l'Environnement (Cuba), Robert T. Watson de la Banque mondiale et de David Jon Dokken of University Corporation for Atmospheric Research ; Avril 2002, 89 p.

18. GIEC, 2006. Guide pour l'inventaire national des gaz à effet de serre agriculture, foresterie et autre usage des terres. Institute for Global Environnemental Strategies, Japon 4: 6 p.

19. GIEC, 2007. Bilan 2007 des changements climatiques. Contribution des Groupes de travail I, II et III au quatrième Rapport d'évaluation du Groupe d'experts intergouvernemental sur l'évolution du climat [Équipe de rédaction principale, Pachauri, R.K. et Reisinger, A., Genève, Suisse, 103 p.

20. Gockowski and Sonwa. D., 2011. Cocoa Intensification Scenarios and Their Predicted Impact on $\mathrm{CO}^{2}$ Emissions, Biodiversity Conservation, and Rural Livelihoods in the Guinea Rainforest of WestAfrica, $12 \mathrm{p}$.

21. Gonmadjé C.F., Doumenge C., McKey D., Tchouto M.G.P., Sunderland T.C.H., Balinga M.P.B., Sonké B., 2011. Tree diversity and conservation value of the Ngovayang massif, Cameroon. Biodiversity and Conservation 20: 2627-2648. http://dx.doi.org/10.1007/s10531011-0095-z. 
22. Gonmadjé C.F., Doumenge C., Sunderland T.C.H., Balinga M.P.B., Sonké B., 2012. Analyse phytogéographique des forêts d'Afrique Centrale : le cas du massif de Ngovayang (Cameroun). Plant Ecology and Evolution 145 (2): 152-164, 2012, 13 p.

23. Hamawa, Y., 2005. Caractérisations biophysiques des jardins de case chez les populations de Niza'a (Adamaoua, Cameroun). Mémoire de DEA, Université de Yaoundé I, 71 p.

24. Jagoret, P., Michel-Dounias, I., Malézieux, E., 2011. Long-term dynamics of cocoa agroforests: a case study in central Cameroon. Agroforest Syst (2011) 81:267-278, springer Science + Business Media B.V. 2011, 12 p.

25. Jagoret, P., Michel-Dounias, I., Snoeck, D., Ngnogué, T., Malézieux, E., 2012. Afforestation of savannah with cocoa agroforestry systems: a small-farmer innovation in central Cameroon. Agroforest Syst (2012) 86:493-504, springer Science + Business Media B.V. 2012, 12 p.

26. Jiofack, T., Guedje, N.M., Tchoundjeu, Z., Fokunang, C., Lejoly, J., Kemeuze, V., 2013. Agroforestry typology of some cocoa based agroforests in the Mbam and Inoubou division: The importance for local population livelihoods. Journal of Ecology and the Natural Environment, ISSN 2006-9847 (C2013 Academic Journals, 9 p.

27. Kacholi, D.S., 2014. Analysis of Structure and Diversity of the Kilengwe Forest in the Morogoro Region, Tanzania International Journal of Biodiversity. Volume 2014, Article ID 516840, 8 p.

28. Kengne Fodouop et Ali A. Jong, 2002. Producteurs ruraux dans la crise au Cameroun : la province du Centre, L'Harmattan, 2002, 164 p.

29. Kotto-same J., Zapfack L., et Moukan S., 1997. Carbon dynamics in slash and burn agriculture and land use alternatives in the humid forest zone of Cameroon. Agriculture, Ecosystems and environment, 3 p.

30. Lebrun J.P., Stork A.L., 1991, 1992, 1995, 1997. Enumération des plantes à fleur d'Afrique tropicale, 4 Vols. Genève, Conservatoire et Jardin botaniques de la ville de Genève.

31. Leplaideur A., 1985. Les systèmes agricoles en zone forestière, les paysans du Centre et du sud Cameroun. IRAT, Yaoundé, 615 p.

32. Letouzey, 1985. Notice de la carte phytogéographique du Cameroun au 1: 500.000. 5 documents et 6 cartes. Institut de la Carte Internationale de la Végétation, Toulouse, $8 \mathrm{p}$.

33. Mamah, M. 2007. Contraintes, opportunités environnementales et développement du village idool (Adamaoua - Cameroun). Mémoire de Maitrise. Université de Ngaoundéré, 70 p.

34. Manfo D.A., Tchindjang M., Youta H. J., 2015. Systèmes agroforestiers et conservation de la biodiversité dans un milieu 
fortement anthropisé : le cas d'Obala. Revue Scientifique et Technique Forêt et Environnement du Bassin du Congo, 5.12 p.

35. Mapongmetsem P.M., 2017. Domestication et culture des espèces d'intérêt socio-économique au Cameroun. Lecon inaugurale aux Doctoriales 2017. Univ.Ngaoundéré, Cameroun. Excelence Plus (éd.), $47 \mathrm{p}$.

36. Mapongmetsem, P.M., Mbofung, C., Ibrahima, A., Tchuenguem, F.N., Alexandre, D.Y. \& Mefeng O., 2000. Situation des jardins de case dans les savanes soudano-guinéennes de l'Adamaoua : cas de la zone périurbaine de Ngaoundéré (Cameroun). Bioscience proceeding, 7: 9 p.

37. Mapongmetsem, P.M., Hamawa, Y., Baye-Niwah, C., Froumsia, M., Zigro, L. \& Meiga, O. S., 2009. Conservation et valorisation de la biodiversité dans les agrofôrets de case de la zone SoudanoGuinéenne. In: X. van der Burgt, J. van der Maesen \& J.-M. Onana (eds), Systematics and conservation of African plants, Royal Botanic Gardens, Kew, 9 p.

38. Mapongmetsem P. M., Etchiké D., Ngassoum M. B., 2016. Conservation et valorisation de la biodiversité dans les agroforêts de la zone périurbaine de la ville Bafia (Région du Centre au Cameroun). Revue Scientifique et Technique Forêt et Environnement du Bassin du Congo, $6: 9 \mathrm{p}$.

39. Mbolo, 1998. Rapport de consultation «Écologie -Biodiversité » pour l'Office National de Mc Donald L.H (ed)., 1982. Agroforetry in african humid tropics. United University, Tokyo, $14 \mathrm{p}$.

40. MINADER/DESA, 2005/2006. Présentation des résultats des fiches agricoles du 3ème RGPH. Avec la collaboration du Bureau Central des Recensements et des Etudes de Population (BUCREP) et de l'Institut National de la Statistiques (INS), $120 \mathrm{p}$.

41. Montagnini F., et Nair P.K.R., 2004. Carbon sequestration: An underexploited environmental benefit of agroforestry systems. Agroforestry Systems, 61: $14 \mathrm{p}$.

42. Nair, P. K. R B., Kumar M., and Nair, V.D., 2009. Agroforestry as a strategy for carbon sequestration. Plant Nutr. Soil Sci. 172, 14 p.

43. Ngomanda, A., Lebamba, J., Laurier Engone-Obiang N., Lepengue, N., M'Batchi, B., 2013. Caractérisation de la Biomasse sèche des mosaïques forêt-savane des plateaux Okouma et Bagombé au sud-est du Gabon. Journal of Applied Biosciences, 68 : 5417-5425ISSN 1997$5902,9 \mathrm{p}$.

44. Ngomanda A., Laurier N., Lebamba J., Moundounga Q., Gomat H., Mankou G., Loumeto J., Midoko D., Ditsouga F., Koumba R, Henga K., Okouyi C., Nyangadouma R, Lépengué N., Mbatchi B., Picard N., 
2014. Site-specific versus pantropical allometric equations: Which option to estimate the biomass of a moist central African forest? Forest Ecology and Management 312: 9 P.

45. Norgrove and Hauser., 2013. Carbon stocks in shaded Theobroma cacao farms and adjacent secondary forests of similar age in Cameroon Tropical Ecology 54(1): 2013 International Society for Tropical Ecology www.tropecol.com; 8 p.

46. Ogouwalé E., 2004. Changements climatiques et sécurité alimentaire dans le Bénin méridional. Cotonou : Mémoire de DEA, Université d'Abomey-Calavi (UAC), École Doctorale Pluridisciplinaire (EDP) « Espace, Cultures et Développement », Faculté des Lettres Arts et Sciences Humaines (FLASH), 119 p.

47. Ogouwalé E., 2006. Changements climatiques dans le Bénin méridional et central : indicateurs, scénarios et prospective de la sécurité alimentaire. Cotonou : Thèse de Doctorat, LECREDE/ FLASH/ EDP/ UAC, 302 p.

48. Oyono, P.R. 1999. An overview on farmers' organization and NGOs in the humid forest zone of Cameroon. Some lessons of partnership with research at IITA-HFC. Contribution to CCER visit at IITA/HFC. P. E., 12 p.

49. Saj S., Patrick Jagoret., Herve Todem Ngogue, 2013. Carbon storage and density dynamics of associated trees in three contrasting Theobroma cacao agroforests of Central Cameroon, $7 \mathrm{p}$.

50. Saj, S., Durot, C., Mvondo-Sakouma, K., Tayo Gamo, K., \& Avana Tientcheu, M. L., 2017. Contribution of companion trees to long-term tree conservation, carbon storage and agroforest sustainability: a functional analysis of the diversity in cacao plantations of Central Cameroon. International Journal of Agricultural Sustainability ; 15 p.

51. Salbai, A., 2001. Caractérisation biophysique et socio-économique des jardins de case de Belel (Adamaoua-Cameroun). Mémoire de maîtrise. Université de Ngaoundéré, 68 p.

52. Soh J.C., 1999. Etude des ressources fauniques et de leurs utilisations dans la zone Ndiki - Makénéné. Projet de conservation de la biodiversite dans la vallee de l'inoubou. Rapport CAFER. 64 p.

53. Sonké, B., 1998. Etudes floristiques et structurales des forêts de la Réserve de Faune du Dja (Cameroun) : Thèse de Doctorat, Université Libre de Bruxelles ; 267 p. 
54. Sonké, B., 2004. Environnement biologique du site de Nkamouna ; Biodiversité - Flore. Etude d'impact environnemental du projet minier de GEOVIC à Nkamouna. Rainbow Environment Consult - Août $2004 ; 5$ p.

55. Sonwa, D.J., Weise, S.F., Tchatat, M., Nkongmeneck, A.B., Adesina, A., Ndoye, O. et Gockowski, J. 2000. Les agroforêts cacao : espace intégrant développement de la cacaoculture, gestion et conservation des ressources forestières au Sud-Cameroun. Paper presented at the second Pan African Symposium on The sustainable use of Natural resources in Africa. Ouagadougou, Burkina Fasso, 24-27 July 2000. $124 \mathrm{p}$.

56. Sonwa, D.J, Wandji, D.N.D., Gockowski, J.J, Weise, S.F., Legg, C. 2002. Conservation de la biodiversité et développement d'un système pilote d'information sur la production et la commercialisation dans les agroforêts cacao du Sud-Cameroun. Contribution pour le séminaire organisé par l'OCDE (Organisation de coopération et de développement économique) à Dakar, Sénégal, du 25-27 juin 2002 sur le thème «international workshop on incentives for biodiversity conservation and sustainable use». $15 \mathrm{p}$.

57. Sonwa D., 2004. Biomass management and diversification within cocoa agroforests in the humid forest zone of southern Cameroon. $\mathrm{PhD}$ Thesis. Institüt für Gartenbauwissenschaft der Rheinischen FriedrichWilhelms-Universität Bonn. $112 \mathrm{p}$.

58. Sonwa, D. J., Nkongmeneck, B. A., Weise, S. F., Tchatat, M., Adesina, A. A., \& Jansens, M. J. J., 2007. Diversity of plants in cocoa agroforests in the humid forest zone of Southern Cameroon. Biodiversity and Conservation ; $16 \mathrm{p}$.

59. Tayo Gamo, K. Y., 2014. Dynamique de la biodiversité ligneuse et des stocks de carbone dans les systèmes agroforestiers à base de cacaoyer au centre Cameroun : cas de Ngomedzap. Master professionnel en foresterie, FASA, $77 \mathrm{p}$.

60. Tchatat, M., 1996. Les jardins de case agroforestiers des basses terres humides du Cameroun : étude des cas des zones forestières des provinces du Centre et du Sud. Thèse de Doctorat. Université de Paris II. France, 145 p.

61. Tchotsoua M., Mohamadou G, Esoh E and Ngana J.P., 1998. Diagnostic de l'état de l'environnement de Ngaoundéré et contribution pour une approche de gestion partagée. Annales de la FALSH de Ngaoundéré, Vol 3 : 15 p.

62. Temgoua, L. 2007. Etude préalable à l'aménagement de la réserve forestière de Mbalmayo (Cameroun) : pratiques et modes d'accès des populations locales. Mémoire pour l'obtention du diplôme de Master. 
«Dynamique Spatiale, Gestion des Territoires et Développement » spécialisation : «Acteurs et Nouvelles Territorialités (ANT) »UFR III Septembre $2007 ; 121 \mathrm{p}$.

63. Temgoua L. F., Dongmo W., Nguimdo V., Nguena C., 2018. Diversité Ligneuse et Stock de Carbone des Systèmes Agroforestiers à base de Cacaoyers à l'Est Cameroun : Cas de la Forêt d'Enseignement et de Recherche de l'Université de Dschang. Journal of Applied Biosciences $12 \mathrm{p}$.

64. Temgoua L. F., Momo S. M.C., Boucheké R. K., 2019. Diversité Floristique des Ligneux des Systèmes Agroforestiers Cacaoyers du Littoral Cameroun : Cas de l'Arrondissement de Loum. European Scientific Journal ; March 2019 edition Vol.15, No.9 ISSN: 1857 7881, 62-83, $21 \mathrm{p}$.

65. Traoré, S.A., 1997. Analyse de la flore ligneuse et de la végétation de la zone de Simenti (parc National du Niokolo Koba). Sénégal Oriental. Doctorat de 3eme cycle en Biologie Végétale (Option : Botanique Gestion des Ressources Forestières). Université Cheikh Anta Diop De Dakar ; 147 p.

66. Tsoumou B. R., Lumandé K. J., Kampé J. P., Nzila J. D., 2016. Estimation de la quantité de Carbone séquestré par la Forêt Modèle de Dimonika (Sud-ouest de la République du Congo). Revue Scientifiques et techniques Forêts et Environnement du Bassin du Congo (RIFFEAC), $6: 39-45,7$ p.

67. UICN-PC, 2013. Comment aborder la REDD+ au Cameroun : Contexte, enjeux et options pour une stratégie nationale. Yaoundé, Cameroun : UICN, 103 p.

68. Vissoh Pierre V., Tossou Rigobert C., Houinsou Dedehouanou, Hervé Guibert, Olivier C. Codjia, Vodouhe Simplice D. et Agbossou Euloge K., 2015. Perceptions et stratégies d'adaptation aux changements climatiques : le cas des communes d'Adjohoun et de Dangbo au SudEst Bénin. Les Cahiers d'Outre-Mer, Revue de géographie de Bordeaux ; 260, 15 p.

69. Warwick, R.M., Ashman, C.M. et Brown, A.R., 2002. Inter-annual changes in the biodiversity and community structure of the macrobenthos in Tees Bay and the Tees estuary, UK, associated with local and regional environmental events. Mar Ecol Prog Ser 234 p.

70. White, L. \& Edwards, A. 2001. Conservation en forêt pluviale africaine : Méthodes et recherches. Wildlife conservation society, New york, $444 \mathrm{p}$.

71. Winrock, 2005. Guide de Mesure et de Suivi du Carbone dans les Forêts et Prairies Herbeuses. Winrock International, Timothy Pearson et Sandra Brown, 1621 N., Kent St., Suite 1200 Arlington, VA 22209, 
USA ; 39 p.38. Youta, 1998. Arbres contre graminées : la lente invasion de la savane par la forêt au Centre-Cameroun. Thèse de doctorat nouveau régime. Université de Paris-Sorbonne (Paris IV). U.F.R. de Géographie et Aménagement Spécialité : Biogéographie, $241 \mathrm{p}$.

72. Youta, 1998. Arbres contre graminées : la lente invasion de la savane par la forêt au Centre-Cameroun. Thèse de doctorat nouveau régime. Université de Paris-Sorbonne (Paris IV). U.F.R. de Géographie et Aménagement Spécialité : Biogéographie, $241 \mathrm{p}$.

73. Zapfack, L., 2005. Impact de l'agriculture itinérante sur brûlis sur la biodiversité végétale et la séquestration du carbone en zone forestière. Thèse de doctorat d'Etat. Université de Yaoundé I ; 194 p.

74. Zapfack, L., Weise, S. F., Ngobo, M., Tchamou, N. \& Gillison A., 2000. Biodiversité et produits forestiers non ligneux de trois types de jachères du Cameroun méridional. In : Floret C., Pontanier R., Libbey J. La jachère en Afrique tropicale. Eurotext, Paris $@$, 2000., 8 p. 